\title{
N91-26577
}

\section{Late Wisconsin and Early Holocene Glacial History, Inner Ross Embayment, Antarctica}

\author{
George H. Denton \\ Department of Geological Sciences and Institute for Quaternary Studies, University of Maine, \\ Orono, Maine 04469 \\ James G. Bockheim and Scott C. Wilson \\ Department of Soil Science, University of Wisconsin, Madison, Wisconsin 53706
}

AND

Minze Stuiver

Quaternary Isotopes Laboratory, Quaternary Research Center, University of Washington. Seattle, Washington 98195

\begin{abstract}
Lateral drift sheets of outlet glaciers that pass through the Transantarctic Mountains constrain past changes of the huge Ross ice drainage system of the Antarctic Ice Sheet. Drift stratigraphy suggests correlation of Reedy III (Reedy Glacier), Beardmore (Beardmore Glacier), Britannia (Hatherton/Darwin Glaciers), Ross Sea (McMurdo Sound), and "younger" (Terra Nova Bay) drifts; radiocarbon dates place the outer limits of Ross Sea drift in late Wisconsin time at 24,00013,000 yr B.P. Outlet-glacier profiles from these drifts constrain late Wisconsin ice-sheet surface elevations. Within these constraints, we give two extreme late Wisconsin reconstructions of the Ross ice drainage system. Both show little elevation change of the polar plateau coincident with extensive ice-shelf grounding along the inner Ross Embayment. However, in the central Ross Embayment one reconstruction shows floating shelf ice, whereas the other shows a grounded ice sheet. Massive late Wisconsin/Holocene recession of grounded ice from the western Ross Embayment, which was underway at 13,040 yr B.P. and completed by $6600-6020$ yr B.P., was accompanied by little change in plateau ice levels inland of the Transantarctic Mountains. Sea level and basal melting probably controlled the extent of grounded ice in the Ross Embayment. The interplay between the precipitation (low late Wisconsin and high Holocene values) and the influence of grounding on outlet glaciers (late Wisconsin thickening and late Wisconsin/Holocene thinning, with effects dying out inland) probably controlled minor elevation changes of the polar plateau. 01989 University of Washington.
\end{abstract}

\section{INTRODUCTION}

The Antarctic Ice Sheet is one of the most prominent physical features on our planet. A knowledge of its past behavior can help solve the cause of late Quaternary ice ages. For example, Antarctic ice-sheet reconstructions form part of global snapshots of the last glacial maximum that are a basis for atmospheric modeling experiments. In the pinpointing of physical mechanisms of paleoclimate changes, the influence of the Antarctic Ice Sheet (including potential fringing shelf ice) on ice-age cli- mates can be determined by prescribing reconstructed ice-sheet geometry as the sole variable input into global climate modeling experiments that compare ice-age and modern climates. This reconstructed geometry can also give the contribution of Antarctic ice to sea-level and marine oxygen isotope records. A knowledge of Antarctic icevolume changes constrains interpretations of marine oxygen isotope records and, hence, Northern Hemisphere ice-sheet history. Finally, the paleoclimate record from this far-southern region is an important part of the global data bank used to test ice-age 
theories, particularly with respect to the relative timing of climate changes between polar hemispheres.

To address these issues, we describe here late Quaternary paleoclimate, icevolume change, and dynamics of the Ross ice drainage system using data from a powerful combination of drift sheets and ice cores. The Ross ice drainage system encompasses about one-fourth of the surface area of the Antarctic Ice Sheet (Fig. 1). Inland ice divides encircling this drainage system are $5700 \mathrm{~km}$ long and display numerous domes and saddles. Ice from the high East Antarctic plateau flows seaward in outlet glaciers that pass through the Transantarctic Mountains into the Ross Ice Shelf or Ross Sea. Ice from the marine-based West
Antarctic ice sheet flows through major ice streams into the Ross Ice Shelf. Three deep ice cores with dated paleoclimatic records come from within the Ross ice drainage system (Jouzel et al., 1989). Drift sheets occur alongside Transantarctic Mountain outlet glaciers deep within the Ross Embayment.

We start by selecting Transantarctic Mountains outlet glaciers that lie along ice flowlines leading inland to major domes and ice-core sites. From lateral drift sheets we then reconstruct former longitudinal icesurface profiles of these outlet glaciers. For age control of these profiles, we develop a numerical chronology for several drift sheets in the Taylor Valley/McMurdo Sound and Hatherton/Darwin Glacier areas

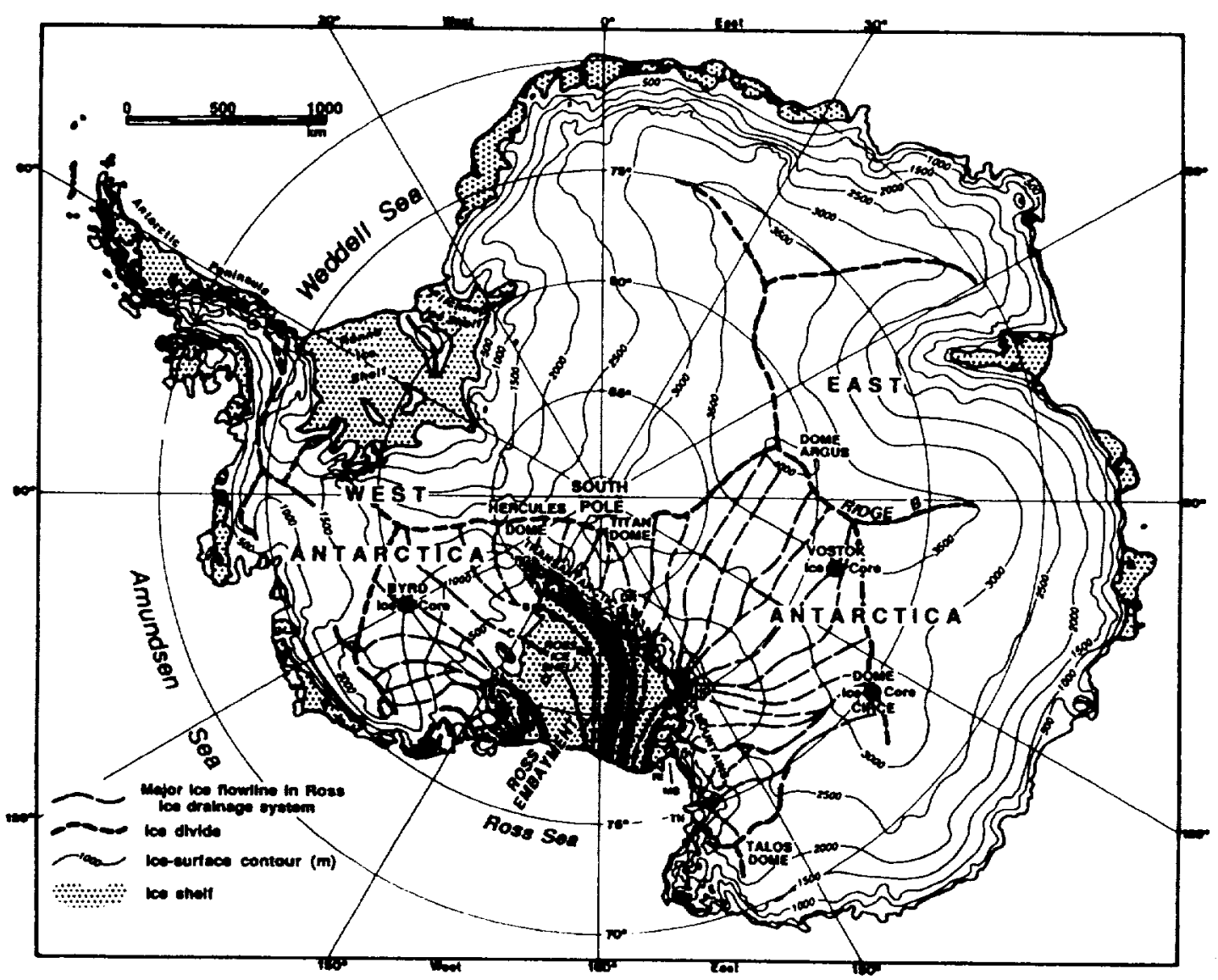

Fic. I. Index map of Antarctica. Flowlines shown only for the Ross ice drainage system, which is defined as the portion of the Antarctic Ice Sheet that flows into the Ross Embayment. A,B,C,D, and $E$ are West Antarctic ice streams. RG, Reedy Glacier; BG. Beardmore Glacier; DR, Dominion Range ice cap; HG, Hatherton Glacier; DG, Darwin Glacier; DV, Dry Valleys; RI, Ross Island; MS, McMurdo Sound; and TN, Terra Nova Bay. Adapted from Drewry (1983). 
(Fig. 1). This numerical chronology is then supplemented by a relative chronology that employs surface boulder weathering and soil development to correlate drift sheets and, hence, ice profiles among the selected outlet glaciers. The late Wisconsin longitudinal profiles of these selected Transantarctic outlet glaciers constrain elevation changes of the inland ice plateau through the last glacial cycle. Hence, they bear directly on Antarctic ice-volume changes as well as on the interpretation of paleotemperatures from interior ice cores.

The outlet glacier profiles also bear on the configuration of grounded and floating ice in the marine portion of the Ross ice drainage system. This problem has been of interest for nearly $150 \mathrm{yr}$. In 1841 and 1842 , Ross (1847) mapped the edge of the Ross Ice Shelf, or the "Great Icy Barrier." Scott (1905, Vol. 2, pp. 422-425) postulated that "when the Southern glaciation was at a maximum . . . the Great Barrier was a very different formation from what it is today ... the huge glacier, no longer able to float on a sea of 400 fathoms, spread out over the Ross Sea, completely filling it with an immense sheet of ice." Scott (1905, Vol. 2, p. 425) further postulated that, during recession, the ice sheet became buoyant and broke away gradually so that the "... Barrier is the remains of the great icesheet." David and Priestley (1914, Fig. 46, PI. XCV) also postulated northward expansion so that at the time of maximum glaciation the surface of the grounded Ross Ice Barrier (Ross Ice Shelf) reached heights of $1000 \mathrm{ft}(305 \mathrm{~m})$ in McMurdo Sound and filled the Ross Sea with a grounded "great ice sheet" for at least $320 \mathrm{~km}$ north of the current Barrier (Shelf) edge. In sharp contrast, Debenham (1921, Fig. 11) interpreted glacial deposits in the McMurdo Sound area in terms of local glaciation. In recent years this difference of opinion concerning extensive (Denton and Armstrong, 1968; Denton et al., 1970, 1971, 1975; Denton and Borns, 1974; Mercer, 1968, 1972; Kellogg et al., 1979) as opposed to restricted (Péwé, 1960; Fillon, 1975) grounded ice in the Ross
Sea has resulted in differing reconstructions of late Wisconsin ice extent in the Ross Embayment. The CLIMAP maximum reconstruction is based largely on geological data and shows widespread grounded ice in the Ross Embayment (Stuiver et al., 1981, pp. 376-380). The CLIMAP minimum reconstruction (Stuiver et al., 1981, p. 375) shows no change in the Ross Embayment and is based on glaciological data that imply little change in the West Antarctic Ice Sheet during the last glacial cycle (Whillans, 1976). In an attempt to reconcile the geologic and glaciologic data, Drewry (1979) reconstructed a restricted complex of local domes and a large floating ice shelf that extends deep into the Ross Embayment to the Transantarctic Mountains at the mouth of major East Antarctic outlet glaciers. Our new ice-surface profiles from the Transantarctic Mountains show deficiencies in all these existing reconstructions. As a result we give here new reconstructions of the minimum and maximum extent of grounded ice in the Ross Embayment permitted by our glacial geologic data in the adjacent Transantarctic Mountains.

\section{LATE WISCONSIN SUBSTAGE}

\section{Ice-Surface Profiles}

Figures 2, 3, and 4 show former longitudinal profiles of late Quaternary age for selected outlet glaciers of the East Antarctic Ice Sheet that flow through the Transantarctic Mountains to the Ross Ice Shelf. These selected outlet glaciers are among the few in the Transantarctic Mountains that have late Quaternary lateral drift sheets. Reedy Glacier lies along a flowline that leads from the Ross Ice Shelf inland through Ice Stream A to the interior Hercules Dome (Fig. 1). Beardmore Glacier is on a flowline that projects inland to the Titan Dome. The Hatherton Glacier is tributary to the Darwin Glacier, which drains ice from Dome Circe.

Former longitudinal profiles were derived from the outer limits of drift sheets correlated among ice-free areas adjacent to 


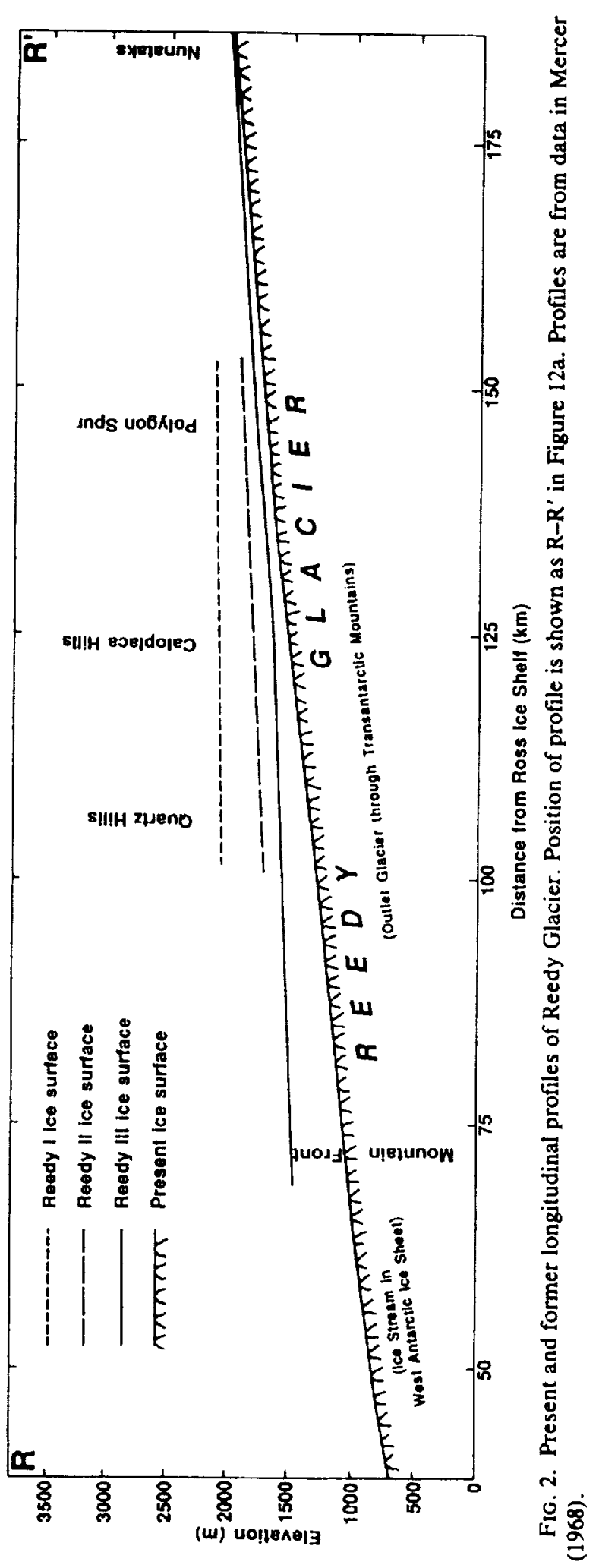




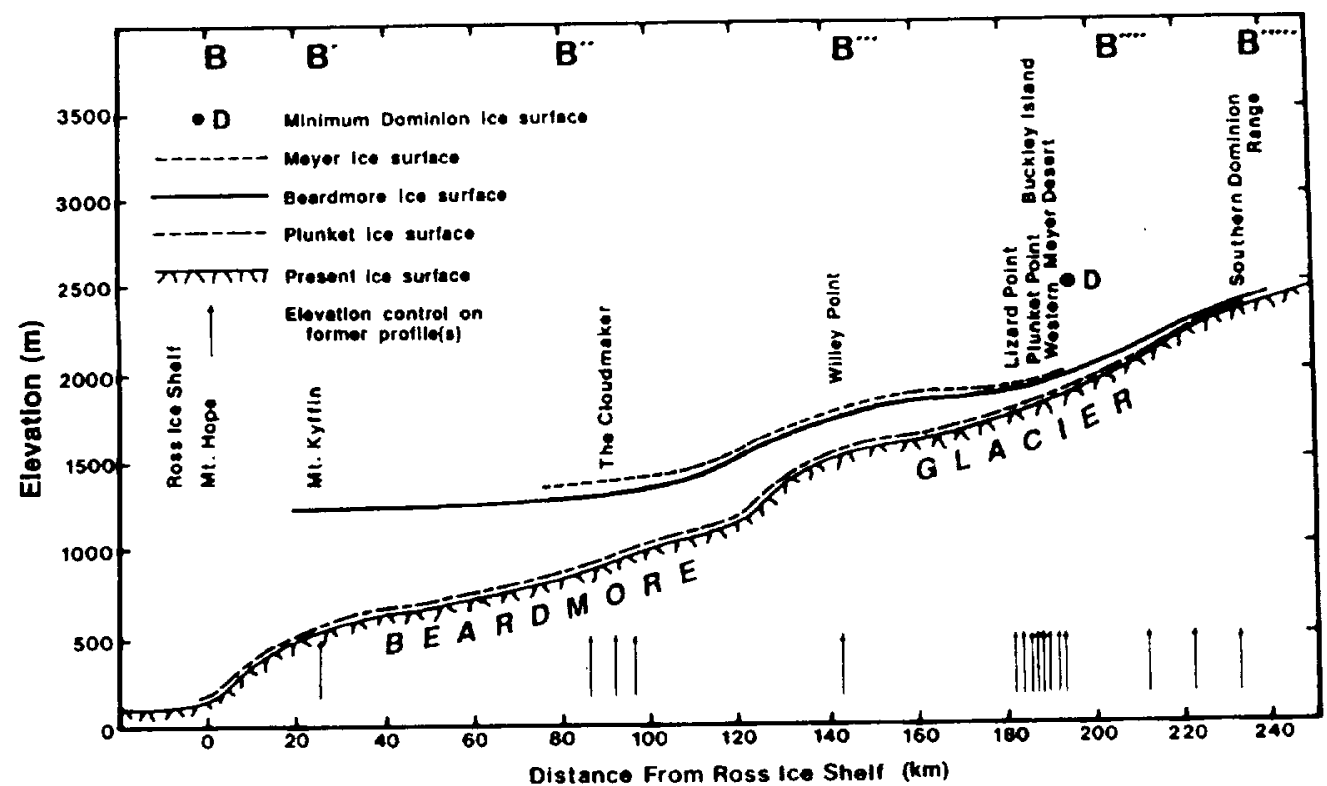

FIG. 3. Present and former longitudinal profiles of Beardmore Glacier. Position of profiles shown as B-B'"'’' in Figure 12a. See Denton et al. (1989) for details.

these outlet glaciers. Details for the Beardmore and Hatherton/Darwin Glacier areas are reported by Denton et al. (1989) and Bockheim et al. (1989). Drift sheets in these two areas were differentiated by surface morphology, surface boulder weathering, and soil development (staining and cohesion depths, solum thickness, morphogenetic salt stage, and weathering stage). We reconstructed former profiles of Reedy Glacier from data in Mercer (1968). We are confident that these former profiles of Reedy Glacier are directly comparable to our profiles of Beardmore and Hatherton Glaciers, because our mapping of drift units at Beardmore Glacier (Denton et al., 1989) afforded results very similar to those obtained in the same field area earlier by Mercer (1972).

Critical to our discussion are the Reedy II and III profiles of Reedy Glacier (Fig. 2) (Mercer, 1968); the Beardmore and Meyer profiles of Beardmore Glacier (Fig. 3) (Denton et al., 1989); and the Britannia I, Britannia II, and Danum profiles of Hatherton Glacier (Fig. 4) (Bockheim et al., 1989). All of these profiles show only slight thickening at glacier heads near the polar plateau of the
East Antarctic Ice Sheet, contemporaneous with substantial thickening at glacier mouths near the Ross Ice Shelf. Our leading interpretation of these ice profiles (Denton et al., 1989; Bockheim et al., 1989) is that they reflect ice-shelf grounding in the southwestern and southern Ross Embayment. An alternative explanation of increased ice flow from higher precipitation on the polar plateau is less plausible. The maximum potential elevation increase along flowlines on the polar plateau (Fig. 1) would be $30 \mathrm{~m}$ inland of Reedy Glacier during the Reedy III episode, $35-40 \mathrm{~m}$ inland of Beardmore Glacier during the Beardmore episode, and $100 \mathrm{~m}$ inland of Hatherton and Darwin Glaciers during the Britannia II episode. From drift geometry, we argued that the Dominion Range ice cap near the polar plateau was retracted when Beardmore Glacier stood at its expanded Beardmore profile (Denton et al., 1989). A strong inference is that precipitation was lower at that time on the Dominion Range ice cap and the adjacent polar plateau. If this inference is correct, the actual elevation change along the polar plateau flowline inland of Beardmore Glacier would depend on the inter- 


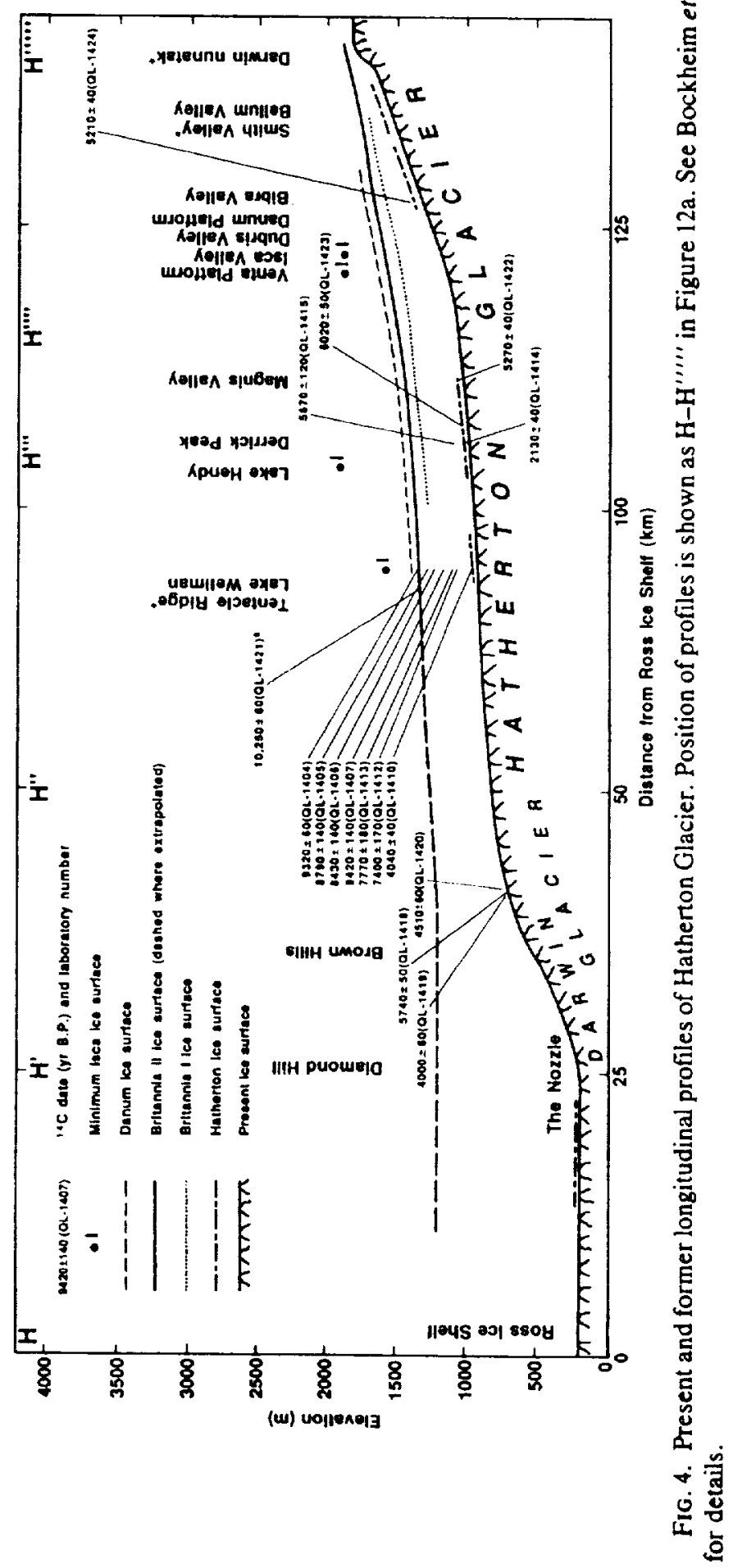


play between the grounding effect and the lowered precipitation. Decreased precipitation could result from lower air temperatures and/or more-distant open water.

The McMurdo Sound/Dry Valleys area is unique in the Transantarctic Mountains for its extensive ice-free terrain. Only here do East Antarctic outlet glaciers fail to reach the Ross Embayment either today or during late Quaternary glaciations. Rather, East Antarctic ice is blocked by the Royal Society Range or, in the case of Taylor Glacier, terminates in an ice-free valley. In the absence of East Antarctic outlet glaciers, late Quaternary ice sheets grounded in the Ross Sea infilled McMurdo Sound and spilled onto peripheral ice-free areas in the Transantarctic Mountains (Fig. 5). Westwardflowing ice lobes from these grounded ice sheets plugged the eastern ends of valleys that open onto the west coast of McMurdo Sound, including Taylor, Marshall, and Miers Valleys. Taylor Valley is particularly important, because it alone among these ice-free valleys contains an East Antarctic outlet glacier. Taylor Glacier, which terminates in the western end of the valley, originates in the peripheral McMurdo Dome on the East Antarctic plateau (Fig. 8). In turn, the small McMurdo Dome, which is controlled by subglacial bedrock, lies at the eastern end of an ice divide and flowline that extend inland to Dome Circe. Hence, the Taylor Valley geological record reveals the relative phasing of the McMurdo Dome and grounded ice sheets in the Ross Sea.

We now discuss three important drift sheets in the McMurdo Sound/Taylor Valley area. Two were deposited by ice sheets grounded in McMurdo Sound and one was deposited by an expanded Taylor Glacier. Ross Sea drift, the most prominent, represents the youngest infilling of McMurdo Sound by grounded ice and is described in detail by Stuiver et al. (1981, pp. 322-355). Figure 5 shows a reconstruction of the grounded Ross Sea ice sheet in McMurdo Sound. The ice-sheet limit in Figure 5

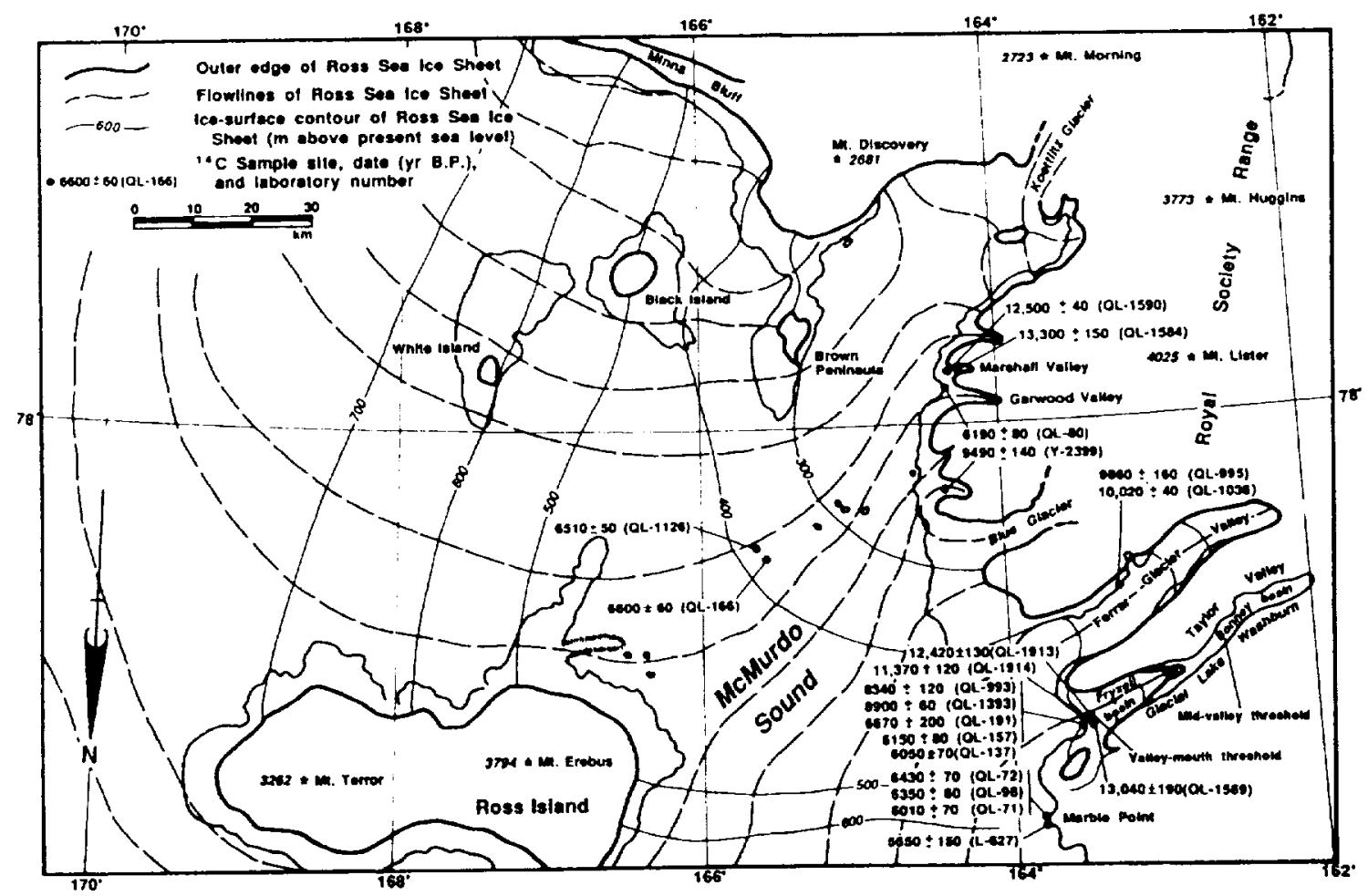

FIG. 5. Surface contours and flowlines of the grounded ice sheet that deposited Ross Sea drift in McMurdo Sound during late Wisconsin (stage 2) time. Adapted from Stuiver et al. (1981, Figs. 7-16). 


\section{ORIGINAL PAGE \\ BLACK AND WHITE PHOTOGRAPH}

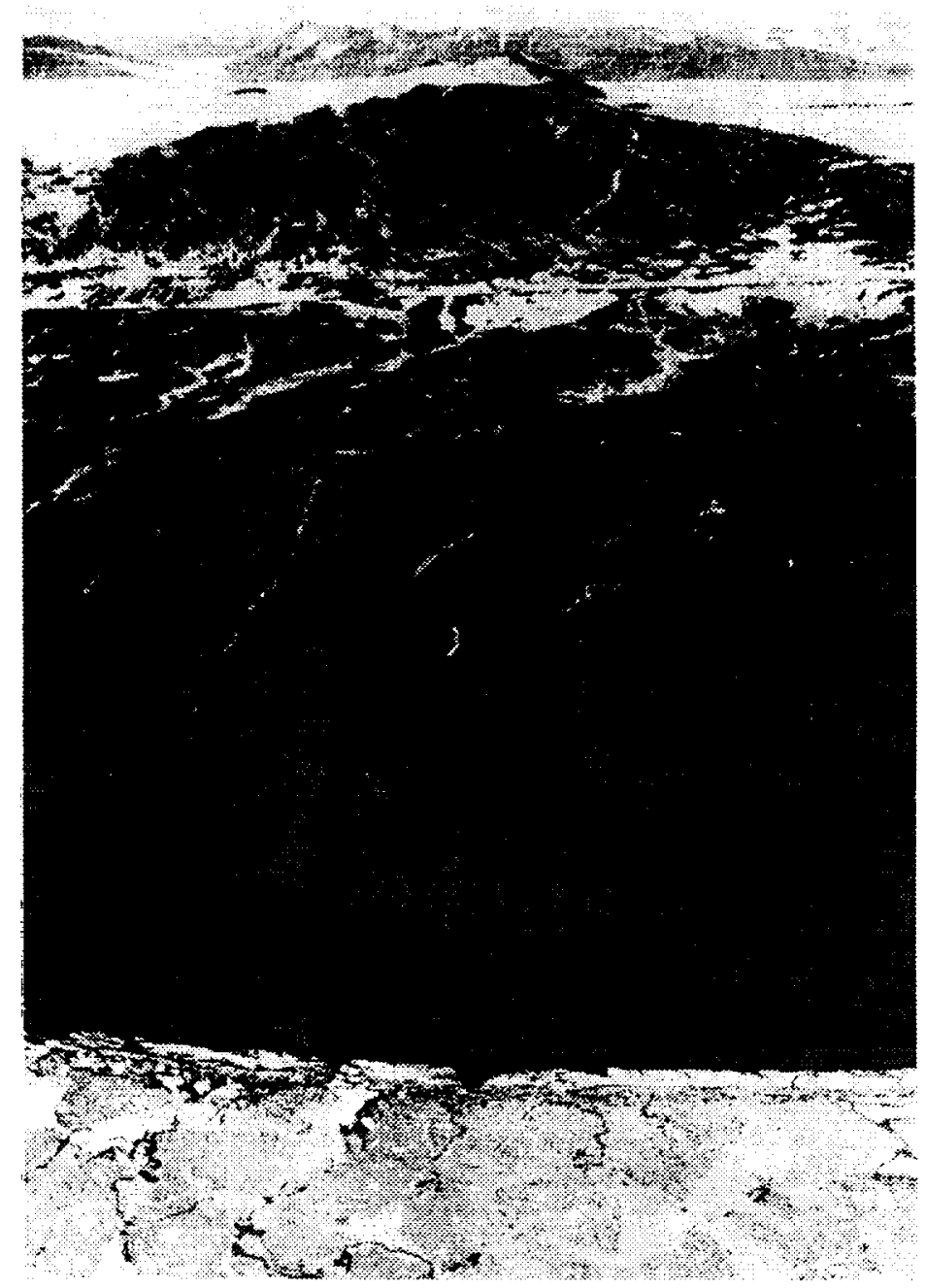

Fig. 6. Oblique aerial view of Ross Sea drift at the mouth of Taylor Valley. The lower part of the photograph shows sea ice on Explorers Cove.

comes from the geologic map depicted in Figure 7-3 of Stuiver et al. (1981). The surface contours are based on measured elevations of the drift limit, typical examples of which are shown in Figures 6 and 7. The former ice flowlines are perpendicular to the ice-surface contours and are consistent with the trend of debris bands and the distribution of distinctive erratics. Marshall drift, the second of these three drifts, is best exposed in Marshall Valley (Fig. S) (Judd, 1986; Dagel et al., 1989). The outer limit of Marshall drift is distal to and parallel with the Ross Sea drift limit along the coastal foothills and valleys of the Royal Society Range on the west coast of McMurdo Sound. From this configuration, as well as from the distribution of distinctive erratics, Dagel et al. (1989) inferred that Marshall drift represents the penultimate infilling of McMurdo Sound by a grounded ice sheet. The third important drift sheet in our discussion is Bonney drift, which occurs on the floor of Taylor Valley and in ice-free areas alongside Taylor Glacier. Bonney drift represents the penultimate advance of Taylor Glacier. It has correlatives alongside most alpine glaciers in Taylor Valley and elsewhere in the McMurdo Sound/Dry Valleys area. Figure 8 shows longitudinal profiles of the former Bonney and Ross Sea ice lobes in Taylor Valley based on the outer limits of the respective drift sheets.

From the reconstruction in Figure 5, we 


\section{ORIGINAL PAGE \\ BLACK AND WHITE PHOTOGRAPH}

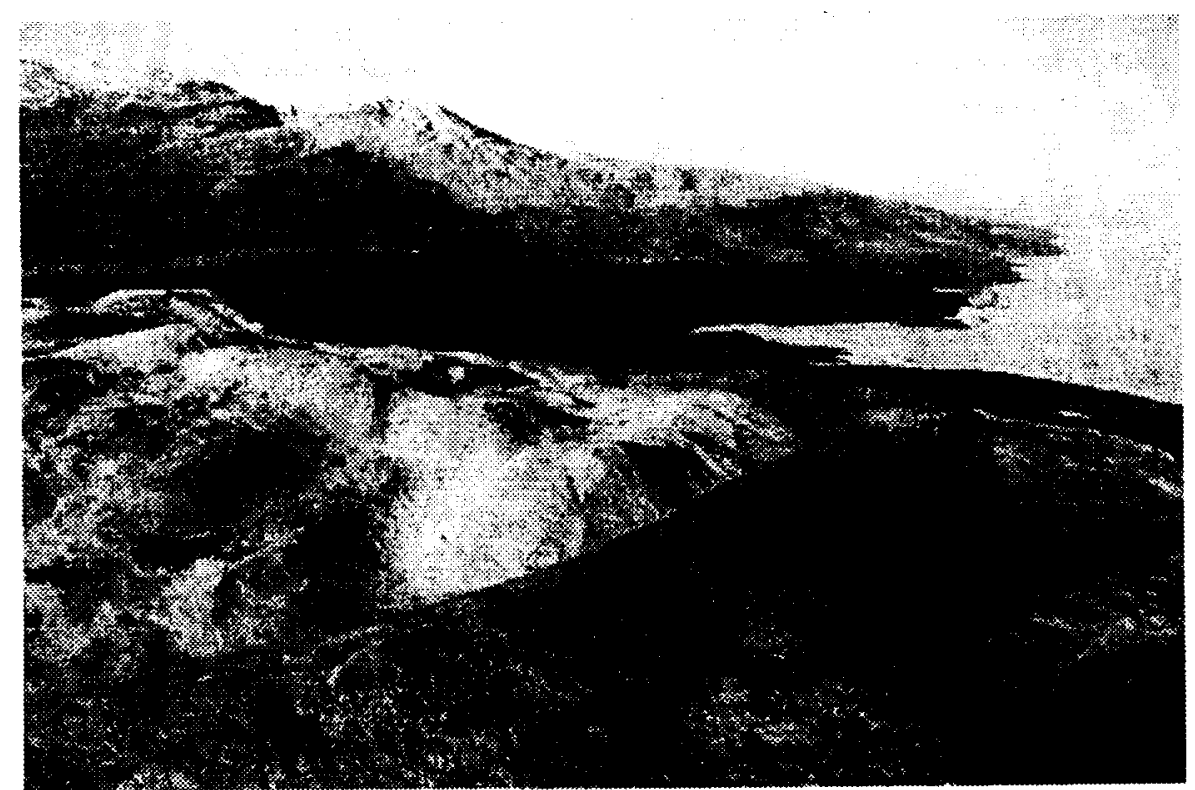

FIG. 7. Oblique aerial view of the upper limit of Ross Sea drift on the west coast of McMurdo Sound near the mouth of Miers Valley.

infer that the Ross Sea and Marshall drifts represent regional ice-sheet grounding, with ice flow from the eastern Ross Sea infilling McMurdo Sound in the absence of through-flowing East Antarctic outlet glaciers. In contrast, we attribute the Bonney advance to local climatic change rather than to ice-sheet grounding in the Ross Sea and McMurdo Sound. We do so for several reasons. First, the Bonney profile indicates that Bonney ice was not contiguous or contemporaneous with a grounded Ross Sea ice sheet in McMurdo Sound (Fig. 8). Hence, Bonney ice does not exhibit the enormous thickening near the Ross Sea shown by the outlet glaciers that drained directly into grounded ice in the Ross Embayment. Second, the Bonney advance was part of a regional expansion that simultaneously involved numerous local alpine glaciers as well as Taylor Glacier. This is distinctly different from the situation that occurred during the last grounding episode when local glaciers receded in the McMurdo Sound/Taylor Valley area (Stuiver et al., 1981). Not only did Bonney drift antedate Ross Sea drift in central Taylor Valley, as shown by overlapping drifts (and hence longitudinal ice profiles) in central Taylor Valley (Fig. 8), but it directly abuts
Ross Sea drift without the intervention of another drift sheet. This geometry almost certainly means that Bonney drift is intermediate in age between Ross Sea and Marshall drifts, which are contiguous on headlands along the west coast of McMurdo Sound. Such an age relationship implies that the Bonney advance was out-of-phase with the last two grounding episodes in McMurdo Sound and the adjacent Ross Sea. Numerical chronologies discussed below confirm these relative ages.

Figure 7-22 in Stuiver et al. (1981) shows two late Quaternary drift sheets at Terra Nova Bay in the northwestern Ross Embayment. Both drifts reflect thickening of at least the lower portions of local outlet glaciers, accompanied by ice-sheet grounding in Terra Nova Bay. Along the outer coast of Terra Nova Bay the upper surface of this grounded ice sheet reached $360 \mathrm{~m}$ during the younger grounding episode and 670-680 $m$ during the older episode.

\section{Chronology}

Numerical chronology. To reconstruct the past behavior of the Ross ice drainage system, we correlate former ice profiles along the length of the Transantarctic Mountains by using our drift stratigraphy. 
First, we develop a numerical chronology of Ross Sea, Marshall, and Bonney drifts in the McMurdo Sound/Taylor Valley area and give minimum ages for Britannia drifts in the Hatherton/Darwin Glacier area. We then apply this numerical chronology to a relative chronology based on drift sequence, surface morphology, and soil development. This relative chronology is the basis for correlation of drift sheets (and hence former longitudinal profiles) among outlet glaciers along the length of the Transantarctic Mountains. These former ice surface profiles allow reconstruction of the Ross ice drainage system at the height of late Wisconsin (Stage 2) glaciation.

An important part of our ${ }^{14} \mathrm{C}$ chronology comes from a unique lacustrine facies of Ross Sea drift. On the west coast of McMurdo Sound, the Ross Sea drift limit is sharp and commonly marked by a moraine ridge on headlands between ice-free valleys. In contrast, Ross Sea drift in valley floors has an irregular outer limit marked in places by near-horizontal terraces. This situation occurs because Ross Sea ice lobes in these valleys terminated in proglacial lakes. At the height of Ross Sea glaciation, interlocked glacial-and-lake-ice systems formed conveyer belts that transported drift from glacial lobes westward into the ice-free valleys on rafts of lake ice (Clayton-Greene, 1986; Clayton-Greene et al., 1988a). During transit, fine-grained sediment passed through lake ice to form stratified lacustrine deposits on lake floors. Coarse sediment and clasts either dropped through fissures in lake ice or were deposited in moats at lake edges. The result was near-horizontal lake-edge terraces and a surficial mantle of valley-wall and lake-floor sand and gravel, some in the form of mounds and ridges. By this mechanism, most Ross Sea drift on valley floors is glacial lacustrine sediment deposited while ice tongues plugged valley mouths and supplied drift to contiguous lake ice. ${ }^{14} \mathrm{C}$ samples are plentiful in such glacial lacustrine drift in the form of bluegreen algae and of carbonate beds.

A suite of ${ }^{14} \mathrm{C}$ dates comes from glacial lacustrine drift in Miers Valley, where a landward flowing Ross Sea ice lobe dammed Glacial Lake Trowbridge (Clayton-Greene, 1986). The ${ }^{14} \mathrm{C}$ dates pinpoint the age of the Ross Sea ice dam in several ways. First, Glacial Lake Trowbridge could not have existed in Miers Valley without an ice dam consisting of a Ross Sea glacier lobe in the mouth of Miers Valley. Further, most of the ${ }^{14} \mathrm{C}$-dated carbonate beds are covered by erratics and gravel hummocks rich in McMurdo volcanics transported inland from the Ross Sea glacier lobe by the lake-ice raft (Clayton-Green, 1986). Hence, these deposits record the existence of a Ross Sea glacier lobe in the valley mouth. Fourteen dates of carbonate and one of blue-green algae all fall between 10,300 \pm $900 \mathrm{yr}$ B.P. (WK-718) and $22,950 \pm 360 \mathrm{yr}$ B.P. (WK-609), hence encompassing most of late Wisconsin time (Clayton-Greene et al., 1988b).

Glacial Lake Washburn occupied the Bonney and Fryxell basins of Taylor Valley during the entire span of late Wisconsin time (Fig. 8) (Stuiver et al., 1981). Two suites of ${ }^{14} \mathrm{C}$ dates of Glacial Lake Washburn lacustrine sediments show when a Ross Sea glacier lobe occupied eastern Taylor Valley. The first suite comes from blue-green algae in deltas of Glacial Lake Washburn. In the Fryxell basin, former lev-

FIG. 8. Longitudinal profiles of present and former glaciers in Taylor Valley. Position of profile is shown as T-T"'."' in Figure 12a. "Taylor I," "Taylor II," and "Taylor III" are temporary names for drift sheets alongside upper Taylor Glacier. "Taylor II" drift corresponds with Bonney drift in Taylor Valley. Inset map of McMurdo Dome is from Drewry $(1980,1982)$. The locations of the ${ }^{14} \mathrm{C}$ dates are schematic because they are projected onto the longitudinal profile from their position on the valley floor and walls. ${ }^{14} \mathrm{C}$ dates listed above the diagram designate deltas in Glacial Lake Washburn; those below the diagram designate Ross Sea glacial lacustrine drift. 


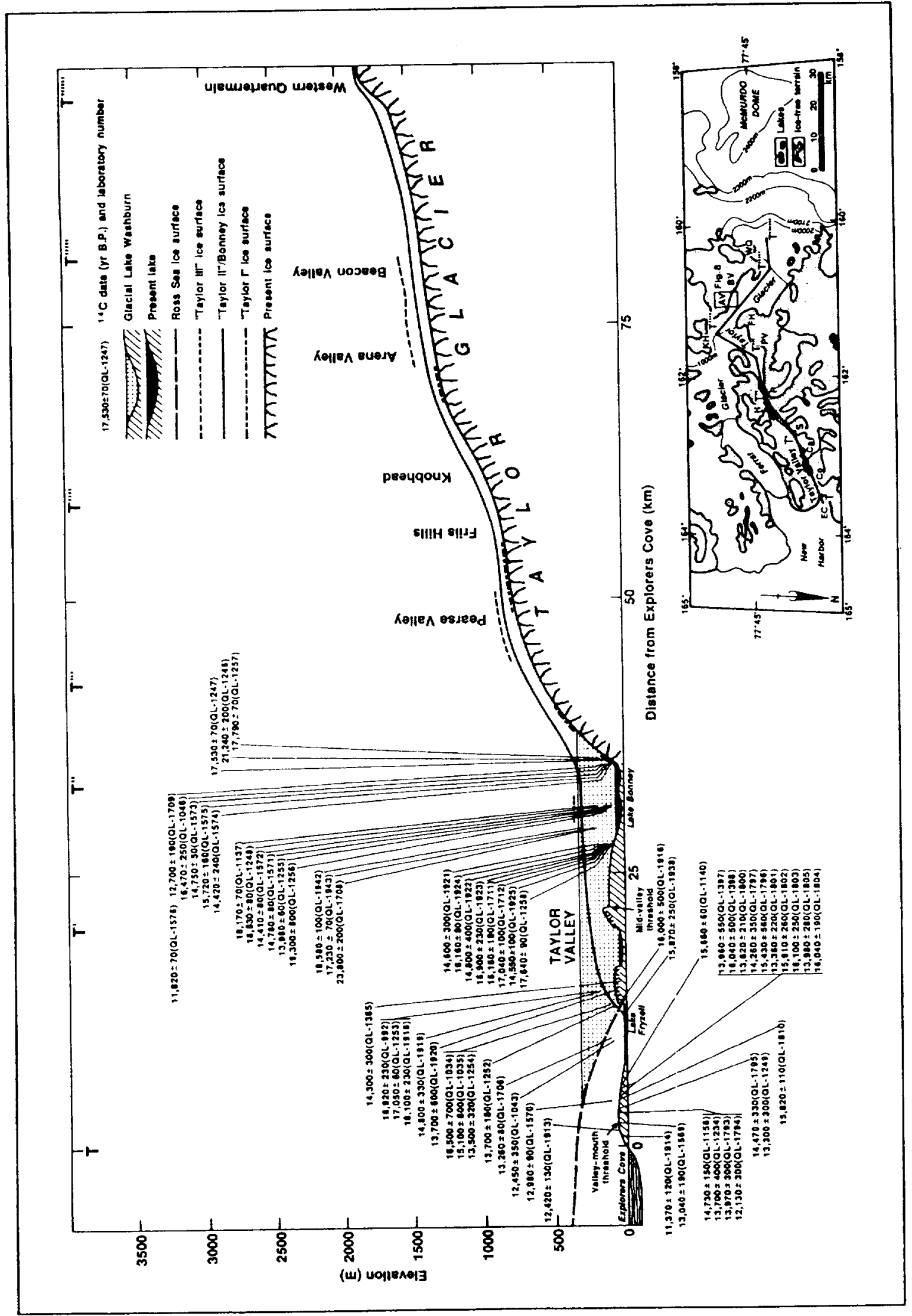


els of Glacial Lake Washburn higher than the valley-mouth threshold, and in the Bonney basin higher than the mid-valley threshold, require a Ross Sea ice dam in eastern Taylor Valley. Details appear in Stuiver et al. (1981, pp. 345-355). Table 1 and Figures 8 and 9 display $32{ }^{14} \mathrm{C}$ dates of perched deltas in Taylor Valley that demonstrate such high lake levels. These ${ }^{14} \mathrm{C}$ dates all fall between $11,820 \pm 70$ yr B.P. (QL-1576) and $23,800 \pm 200$ yr B.P. (QL-1708). The second suite of ${ }^{14} \mathrm{C}$ dates comes from bluegreen algae in glacial lacustrine drift on the eastern valley floor in the Fryxell and Explorers Cove basins. This drift was deposited by the conveyor-belt mechanism in which detritus from the Ross Sea ice lobe was transported into the Fryxell basin on the ice raft of Glacial Lake Washburn. Algae samples from such drift yielded $22{ }^{14} \mathrm{C}$ dates between $11,370 \pm 120$ yг B.P. (QL1914) and $16,100 \pm 250$ yr B.P. (QL-1803) (Table 1 and Figs. 8 and 9). Together, the two suites of ${ }^{14} \mathrm{C}$ dates are consistent in indicating that a Ross Sea ice lobe plugged the mouth of Taylor Valley for at least most of Late Wisconsin (Stage 2) time between 23,800 and 12,450 yr B.P. In addition, the limited number of available ${ }^{14} \mathrm{C}$ dates of glacial lacustrine sediments suggests that the Ross Sea ice lobe projected far into the valley until about 16,000 yr B.P., that the Fryxell basin was largely ice free between 16,000 and 13,300 yr B.P. while the Ross Sea ice lobe terminated on the valleymouth threshold, and that ice recession from the valley-mouth threshold (and hence decline of the ice-sheet surface in McMurdo Sound) was underway by 13,040 yr B.P.

Marshall drift is distal and parallel to Ross Sea drift along the western coast of McMurdo Sound. Its numerical age has been determined in Marshall Valley by 36 U/Th dates of carbonate lacustrine beds within volcanic-rich Marshall drift (Fig. 5) (Dagel et al., 1988; Judd, 1986). All but four of these dates fall between 130,000 and 190,000 yr B.P. Hence, Marshall drift most likely corresponds with Stage 6 in the marine oxygen-isotope record.

There are two sources of numerical dating control on Bonney drift in Taylor Valley. The first is ${ }^{14} \mathrm{C}$ dates of perched deltas of Glacial Lake Washburn in the Bonney basin (Fig. 8, Table 1). These perched deltas are all younger than Bonney drift and therefore their late Wisconsin ${ }^{14} \mathrm{C}$ dates are minimum for this drift. It follows that Bonney drift predates late Wisconsin time and therefore is older than Ross Sea drift in the valley mouth. This confirms the relative ages of these two drifts that was inferred from their crosscutting relationships in central Taylor Valley (Fig. 8). From the areal distribution of ${ }^{14} \mathrm{C}$-dated deltas in the Bonney basin (Fig. 8) it is clear that Glacial Lake Washburn, not Taylor Glacier, occupied much of the Bonney basin throughout late Wisconsin time. The areal distribution and ${ }^{14} \mathrm{C}$ ages of deltas adjacent to its current snout suggest that Taylor Glacier now occupies its maximum position since late Wisconsin time (with the exception of a slight fluctuation that in a few places has left a Holocene ice-cored moraine several meters from the current ice margin). This suggests strongly that the McMurdo Dome on the East Antarctic polar plateau surface inland from Taylor Valley (Fig. 8) was slightly lower than now during late Wisconsin time.

Direct numerical dates of Bonney drift come from carbonates deposited in small lakes dammed in middle Taylor Valley by an expanded Taylor Glacier during its Bonney advance. An extensive program is under way to date these carbonates by the U/Th method. Previously published dates (Hendy et al., 1979) that we now interpret as relating to the Bonney drift are 75,000 \pm $2600,80,000 \pm 2800,92,000 \pm 2000,74,000$ $\pm 1600,95,000 \pm 4500$, and $98,000 \pm 1700$ yr B.P. Hence, Bonney drift most likely corresponds with Stage 5 in the marine oxygen-isotope record.

Farther south in the Transantarctic Mountains at Hatherton Glacier, ${ }^{14} \mathrm{C}$ dates discussed in detail by Bockheim et al. 
TABle 1. Selected ${ }^{14} \mathrm{C}$ Dates from the Taylor Valley/McMurdo Sound Area and the Western ROSS EMBAYMFNT

\begin{tabular}{|c|c|c|c|c|}
\hline $\begin{array}{l}\text { Laboratory } \\
\text { number }\end{array}$ & $\begin{array}{l}{ }^{14} \mathrm{C} \text { date } \\
\text { (yr B.P.) }\end{array}$ & Location & Description & Reference \\
\hline \multicolumn{5}{|c|}{${ }^{14} \mathrm{C}$ dates of Ross Sea drift in eastern Taylor Valley } \\
\hline QL-1914 & $11,370 \pm 120$ & Figure 8 & $\begin{array}{l}\text { Blue-green algae in Ross Sea } \\
\text { glacial laustrine drift }\end{array}$ & This paper \\
\hline QL-1794 & $12,130 \pm 300$ & DO. & DO. & DO. \\
\hline QL-1569 & $13,040 \pm 190$ & DO. & DO. & $\begin{array}{l}\text { DO. } \\
\text { Stuiver et al. }\end{array}$ \\
\hline QL-1249 & $13,300 \pm 300$ & & & $\begin{array}{l}\text { Stuiver et al. } \\
\text { (1981) }\end{array}$ \\
\hline QL-1801 & $13,360 \pm 220$ (AMS) & DO. & DO. & $\begin{array}{l}\text { Denton et al. } \\
\text { (1985) }\end{array}$ \\
\hline $\begin{array}{l}\text { QL-1800 } \\
\text { QL-1234 }\end{array}$ & $\begin{array}{l}13,620 \pm 210 \\
13,700 \pm 400\end{array}$ & $\begin{array}{l}\text { DO. } \\
\text { DO. }\end{array}$ & $\begin{array}{l}\text { DO. } \\
\text { DO. }\end{array}$ & $\begin{array}{l}\text { DO. } \\
\text { Stuiver et al. } \\
\quad(1981)\end{array}$ \\
\hline QL-1397 & $13,960 \pm 550$ & DO. & DO. & $\begin{array}{l}\text { Denton et al. } \\
\text { (1985) }\end{array}$ \\
\hline $\begin{array}{l}\text { QL-1793 } \\
\text { QL-1805 }\end{array}$ & $\begin{array}{l}13,970 \pm 300 \\
13,980 \pm 280\end{array}$ & $\begin{array}{l}\text { DO. } \\
\text { DO. }\end{array}$ & $\begin{array}{l}\text { DO. } \\
\text { DO. }\end{array}$ & $\begin{array}{l}\text { This paper } \\
\text { Denton et al. } \\
\text { (1985) }\end{array}$ \\
\hline QL-1797 & $\begin{array}{l}14,260 \pm 350 \\
14,470 \pm 330\end{array}$ & $\begin{array}{l}\text { DO. } \\
\text { DO. }\end{array}$ & $\begin{array}{l}\text { DO. } \\
\text { DO. }\end{array}$ & $\begin{array}{l}\text { DO. } \\
\text { This paper }\end{array}$ \\
\hline $\begin{array}{l}\text { QL-1795 } \\
\text { QL-1156 }\end{array}$ & $\begin{array}{l}14,470 \pm 330 \\
14,730 \pm 150\end{array}$ & $\begin{array}{l}\text { DO. } \\
\text { DO. }\end{array}$ & $\begin{array}{l}\text { DO. } \\
\text { DO. }\end{array}$ & $\begin{array}{l}\text { This paper } \\
\text { Stuiver et al. } \\
\text { (1981) }\end{array}$ \\
\hline QL-1796 & $15,430 \pm 560$ & DO. & DO. & $\begin{array}{l}\text { Denton ef al. } \\
\text { (1985) }\end{array}$ \\
\hline QL-1140 & $15,660 \pm 60$ & DO. & DO. & $\begin{array}{l}\text { Stuiver et al. } \\
\text { (1981) }\end{array}$ \\
\hline QL-1810 & $15,820 \pm 110$ & DO. & DO. & $\begin{array}{l}\text { This paper } \\
\text { DO. }\end{array}$ \\
\hline QL-1938 & $15,870 \pm 250$ & DO. & DO. & \\
\hline QL-1802 & $15,910 \pm 260(\mathrm{AMS})$ & DO. & DO. & $\begin{array}{l}\text { Denton et al. } \\
\quad(1985)\end{array}$ \\
\hline QL-1916 & $16,000 \pm 500$ & DO. & DO. & This paper \\
\hline QL-1804 & $16,040 \pm 190(\mathrm{AMS})$ & DO. & DO. & $\begin{array}{l}\text { Denton et al. } \\
\text { (1985) }\end{array}$ \\
\hline QL-1398 & $\begin{array}{l}16,040 \pm 500 \\
16,100 \pm 250(\mathrm{AMS})\end{array}$ & $\begin{array}{l}\text { DO. } \\
\text { DO. }\end{array}$ & DO. & DO. \\
\hline \multirow{2}{*}{\multicolumn{5}{|c|}{${ }^{14} \mathrm{C}$ dates of perched deltas of Glacial Lake Washburn in Taylor Valley }} \\
\hline & & & & \\
\hline QL-1043 & $12,450 \pm 350$ & Figure 8 & $\begin{array}{l}\text { Blue-green algae in Glacial Lake } \\
\text { Washburn delta situated in } \\
\text { Fryxell basin above the } \\
\text { elevation of the valley-mouth } \\
\text { threshold }\end{array}$ & $\begin{array}{l}\text { Stuiver el al. } \\
\quad \text { (1981) }\end{array}$ \\
\hline QL-1570 & $12,980 \pm 90$ & DO. & DO. & $\begin{array}{l}\text { Denton et al. } \\
\quad \text { (1985) }\end{array}$ \\
\hline $\begin{array}{l}\text { QL-1706 } \\
\text { QL-1254 }\end{array}$ & $\begin{array}{l}13,260 \pm 80 \\
13,500 \pm 320\end{array}$ & $\begin{array}{l}\text { DO. } \\
\text { DO. }\end{array}$ & $\begin{array}{l}\text { DO. } \\
\text { DO. }\end{array}$ & $\begin{array}{l}\text { DO. } \\
\text { Stuiver et al. } \\
\quad \text { (1981) }\end{array}$ \\
\hline QL-1252 & $13,700 \pm 180$ & DO. & DO. & DO. \\
\hline QL-1920 & $13,700 \pm 600$ & DO. & DO. & This paper \\
\hline QL-1919 & $14,800 \pm 330$ & DO. & DO. & DO. \\
\hline QL-1035 & $15,100 \pm 800$ & DO. & DO. & $\begin{array}{l}\text { Stuiver et al. } \\
\quad(1981)\end{array}$ \\
\hline QL-1034 & $16,500 \pm 700$ & DO. & DO. & DO. \\
\hline QL-992 & $16,920 \pm 230$ & DO. & DO. & DO. \\
\hline QL-1253 & $17,050 \pm 60$ & DO. & DO. & DO. \\
\hline QL-1918 & $18,100 \pm 230$ & DO. & DO. & This paper \\
\hline QL-1385 & $14,300 \pm 300$ & DO. & $\begin{array}{l}\text { Blue-green algae in Glacial Lake } \\
\text { Washburn delta situated in the } \\
\text { Fryxell basin adjacent to the } \\
\text { terminus of Canada Glacier }\end{array}$ & DO. \\
\hline QL-1913 & $12,420 \pm 130$ & DO. & $\begin{array}{l}\text { Blue-green algae in Glacial Lake } \\
\text { Washbum delta on the } \\
\text { valley-mouth threshold }\end{array}$ & DO. \\
\hline QL-1576 & $11,820 \pm 70$ & DO. & $\begin{array}{l}\text { Blue-green algae in Glacial Lake } \\
\text { Washbum delta in the Bonney } \\
\text { basin situated above the } \\
\text { elevation of both the mid-and } \\
\text { valley-mouth thresholds }\end{array}$ & $\begin{array}{l}\text { Denton er al. } \\
\text { (1985) }\end{array}$ \\
\hline
\end{tabular}


TABLE 1-Continued

\begin{tabular}{|c|c|c|c|c|}
\hline $\begin{array}{l}\text { Laboratory } \\
\text { number }\end{array}$ & $\begin{array}{l}{ }^{14} \mathrm{C} \text { date } \\
\text { (yr B.P.) }\end{array}$ & Location & Description & Reference \\
\hline $\begin{array}{l}\text { QL-1709 } \\
\text { QL-1921 } \\
\text { QL-1573 }\end{array}$ & $\begin{array}{l}12,700 \pm 190 \\
14,600 \pm 300 \\
14,750 \pm 50\end{array}$ & $\begin{array}{l}\text { DO. } \\
\text { DO. } \\
\text { Do. }\end{array}$ & $\begin{array}{l}\text { DO. } \\
\text { DO. } \\
\text { DO. }\end{array}$ & $\begin{array}{l}\text { DO. } \\
\text { This paper } \\
\text { Denton et al. } \\
\text { (1988) }\end{array}$ \\
\hline $\begin{array}{l}\text { QL-1922 } \\
\text { QL-1924 } \\
\text { QL-1711 } \\
\text { QL-1577 }\end{array}$ & $\begin{array}{l}14,800 \pm 400 \\
16,160 \pm 80 \\
16,160 \pm 190 \\
16,610 \pm 70\end{array}$ & $\begin{array}{l}\text { DO. } \\
\text { DO. } \\
\text { DO. } \\
\text { DO. }\end{array}$ & $\begin{array}{l}\text { DO. } \\
\text { DO. } \\
\text { DO. } \\
\text { DO. }\end{array}$ & $\begin{array}{l}\text { This paper } \\
\text { DO. } \\
\text { DO. } \\
\text { Denton et al. } \\
\text { (1985) }\end{array}$ \\
\hline QL-1046 & $16,470 \pm 250$ & DO. & DO. & $\begin{array}{l}\text { Stuiver et al. } \\
\quad \text { (1981) }\end{array}$ \\
\hline $\begin{array}{l}\text { QL-1923 } \\
\text { QL-1943 } \\
\text { QL-1257 }\end{array}$ & $\begin{array}{l}16,900 \pm 230 \\
17,230 \pm 70 \\
17,790 \pm 70\end{array}$ & $\begin{array}{l}\text { DO. } \\
\text { DO. } \\
\text { DO. }\end{array}$ & $\begin{array}{l}\text { DO. } \\
\text { DO. } \\
\text { DO. }\end{array}$ & $\begin{array}{l}\text { This paper } \\
\text { DO. } \\
\text { Stuiver et al. } \\
\text { (1981) }\end{array}$ \\
\hline $\begin{array}{l}\text { QL-1137 } \\
\text { QL-1942 } \\
\text { QL-1248 }\end{array}$ & $\begin{array}{l}18,170 \pm 70 \\
18,580 \pm 100 \\
18,830 \pm 80\end{array}$ & $\begin{array}{l}\text { DO. } \\
\text { DO. } \\
\text { DO. }\end{array}$ & $\begin{array}{l}\text { DO. } \\
\text { DO. } \\
\text { DO. }\end{array}$ & $\begin{array}{l}\text { DO. } \\
\text { This paper } \\
\text { Stuiver et al. } \\
\text { (1981) } \\
\text { DO. }\end{array}$ \\
\hline $\begin{array}{l}\text { QL-1246 } \\
\text { QL-1708 }\end{array}$ & $\begin{array}{l}21,240 \pm 200 \\
23,800 \pm 200\end{array}$ & $\begin{array}{l}\text { DO. } \\
\text { DO. }\end{array}$ & $\begin{array}{l}\text { DO. } \\
\text { DO. }\end{array}$ & $\begin{array}{l}\text { Do. } \\
\text { Denton ef al. } \\
\quad(1985)\end{array}$ \\
\hline QL-1255 & $13,980 \pm 60$ & DO. & $\begin{array}{l}\text { Blue-green algae in Glacial Lake } \\
\text { Washburn delta situated in the } \\
\text { Bonney basin below the } \\
\text { elevation of the mid-valley } \\
\text { threshold }\end{array}$ & $\begin{array}{l}\text { Stuiver et al. } \\
\text { (1981) }\end{array}$ \\
\hline$Q L-1572$ & $14,410 \pm 80$ & DO. & Do. & This paper \\
\hline QL-1574 & $14,420 \pm 240$ & DO. & DO. & DO. \\
\hline$Q L-1925$ & $14,550 \pm 100$ & DO. & DO. & DO. \\
\hline QL-1571 & $14,780 \pm 80$ & DO. & DO. & Do. \\
\hline $\begin{array}{l}\text { QL-1575 } \\
\text { QL-1712 }\end{array}$ & $\begin{array}{l}15,720 \pm 160 \\
17,040 \pm 100\end{array}$ & $\begin{array}{l}\text { DO. } \\
\text { DO. }\end{array}$ & DO. & DO. \\
\hline QL-1247 & $17,530 \pm 70$ & DO. & DO. & $\begin{array}{l}\text { Stuiver et al } \\
\text { (1981) }\end{array}$ \\
\hline $\begin{array}{l}\text { QL-1258 } \\
\text { QL-1256 }\end{array}$ & $\begin{array}{l}17,640 \pm 90 \\
19,300 \pm 800\end{array}$ & $\begin{array}{l}\text { DO. } \\
\text { DO. }\end{array}$ & $\begin{array}{l}\text { DO. } \\
\text { DO. }\end{array}$ & $\begin{array}{l}\text { DO. } \\
\text { DO. }\end{array}$ \\
\hline QL-174 & $7020 \pm 60$ & $\begin{array}{l}\text { Dates from the } W \\
\text { Terra Nova Bay. } \\
\text { See Figure } 13\end{array}$ & $\begin{array}{l}\text { Ross Embayment } \\
\text { Adamussium colbecki valves } \\
\text { from recent moraine at edge of } \\
\text { Nansen Ice Sheet }\end{array}$ & $\begin{array}{l}\text { Stuiver ef al. } \\
\quad(1981)\end{array}$ \\
\hline QL-141 & $5340 \pm 50$ & $\begin{array}{l}\text { Franklin Island. } \\
\text { See Figure } 13\end{array}$ & $\begin{array}{l}\text { Lowermost penguin remains in } \\
\text { sea cliff exposure in platform } \\
\text { on west side of Franklin Island }\end{array}$ & DO. \\
\hline QL-71 & $6010 \pm 70$ & $\begin{array}{l}\text { Marble Point. } \\
\text { See Figures } 5 \\
\text { and } 13 .\end{array}$ & Algae in emerged marine delta. & DO. \\
\hline QL-72 & $6430 \pm 70$ & DO. & $\begin{array}{l}\text { Adamussium colbecki valves } \\
\text { from emerged marine delta. }\end{array}$ & DO. \\
\hline $\begin{array}{l}\text { QL-96 } \\
\text { L-627 }\end{array}$ & $\begin{array}{l}6350 \pm 60 \\
5650 \pm 150\end{array}$ & $\begin{array}{l}\text { DO. } \\
\text { DO. }\end{array}$ & $\begin{array}{l}\text { Do. } \\
\text { Elephant seal in emerged beach } \\
\text { deposits. }\end{array}$ & $\begin{array}{l}\text { DO. } \\
\text { Nichols (1968) }\end{array}$ \\
\hline QL-137 & $6050 \pm 70$ & $\begin{array}{l}\text { Explorers Cove, } \\
\text { Taylor Valley. } \\
\text { See Figures } 5 \\
\text { and } 13 .\end{array}$ & $\begin{array}{l}\text { Adamussium colbecki valves } \\
\text { from emerged marine deposits. }\end{array}$ & $\begin{array}{l}\text { Stuiver et al. } \\
\text { (1981) }\end{array}$ \\
\hline $\begin{array}{l}\text { QL-157 } \\
\text { QL-191 }\end{array}$ & $\begin{array}{l}6150 \pm 80 \\
6670 \pm 200\end{array}$ & $\begin{array}{l}\text { DO. } \\
\text { DO. }\end{array}$ & $\begin{array}{l}\text { DO. } \\
\text { Adamussium colbecki valves } \\
\text { from within marine delta. }\end{array}$ & $\begin{array}{l}\text { DO. } \\
\text { DO. }\end{array}$ \\
\hline QL-993 & $8340 \pm 120$ & DO. & $\begin{array}{l}\text { Blue-green algae from lacustrine } \\
\text { delta. }\end{array}$ & DO. \\
\hline QL-1393 & $8900 \pm 60$ & DO. & DO. & $\begin{array}{l}\text { Denton el al. } \\
\text { (1985) }\end{array}$ \\
\hline QL-995 & $9860 \pm 160$ & $\begin{array}{l}\text { Lower Ferrar } \\
\text { Valley. See } \\
\text { Figures } 5 \text { and } \\
13 .\end{array}$ & DO. & $\begin{array}{l}\text { Stuiver et al } \\
\text { (1981) }\end{array}$ \\
\hline
\end{tabular}


TABLE 1 -Continued

\begin{tabular}{|c|c|c|c|c|}
\hline $\begin{array}{l}\text { Laboratory } \\
\text { number }\end{array}$ & $\begin{array}{l}{ }^{14} \mathrm{C} \text { date } \\
\text { (yr B.P.) }\end{array}$ & Location & Description & Reference \\
\hline $\begin{array}{l}\text { QL-1036 } \\
\text { Y-2399 }\end{array}$ & $\begin{aligned} 10,020 & \pm 40 \\
9490 & \pm 140\end{aligned}$ & $\begin{array}{l}\text { DO. } \\
\text { West coast of } \\
\text { McMurdo } \\
\text { Sound. See } \\
\text { Figure } 5 \text {. }\end{array}$ & $\begin{array}{l}\text { DO. } \\
\text { Blue-green algal mat in kettle } \\
\text { lake on ice-cored Ross Sea } \\
\text { drift. }\end{array}$ & $\begin{array}{l}\text { DO. } \\
\text { DO. }\end{array}$ \\
\hline QL-1590 & $12,500 \pm 40$ & $\begin{array}{l}\text { Marshall Valley. } \\
\text { See Figure } 5 .\end{array}$ & $\begin{array}{l}\text { Blue-green algae from surficial } \\
\text { lacustrine sediments on Ross } \\
\text { Sea drif. }\end{array}$ & Dagel et al. (1989) \\
\hline QL-1584 & $13,300 \pm 150$ & DO. & $\begin{array}{l}\text { Blue-green algae in Ross Sea } \\
\text { glacial lacustrine drift. }\end{array}$ & DO. \\
\hline QL-80 & $6190 \pm 80$ & $\begin{array}{l}\text { West coast of } \\
\text { McMurdo } \\
\text { Sound. See } \\
\text { Figures S and } \\
13 \text {. }\end{array}$ & $\begin{array}{l}\text { Blue-green algal mat in kettle on } \\
\text { ice-cored Ross Sea drif. }\end{array}$ & $\begin{array}{l}\text { Stuiver et al. } \\
\quad \text { (1981) }\end{array}$ \\
\hline QL-1126 & $6510 \pm 50$ & $\begin{array}{l}\text { McMurdo Ice } \\
\text { Shelf. See } \\
\text { Figures } 5 \text { and } \\
13 \text {. }\end{array}$ & $\begin{array}{l}\text { Marine shells from surface of } \\
\text { McMurdo Ice Shelf. }\end{array}$ & $\begin{array}{l}\text { Stuiver et al. } \\
\quad \text { (1981) }\end{array}$ \\
\hline QL-166 & $6600 \pm 60$ & DO. & DO. & DO. \\
\hline
\end{tabular}

Note. All dates are conventional dates (Stuiver and Polach, 1977) normalized on $8^{13} \mathrm{C}=-25 \%$. Corrections for seawater ${ }^{14} \mathrm{C}$ deficiency have not been made for dates of marine shells and penguin remains. AMS denotes accelerator mass spectrometry.

(1989) are consistent with the proposition that recession from the Britannia drifts was largely a late Wisconsin/Holocene phenomenon and that recession from Hatherton drift is Holocene in age. From geological considerations, we argued that the Britannia I and II drifts reflect the same major glaciation, with Britannia I drift representing a late readvance superimposed on ice recession from Britannia II drift (Bockheim et al., 1989). If this argument is correct, the implication from the ${ }^{14} \mathrm{C}$ dates is that the Britannia II drift limit represents the late Wisconsin stand of Hatherton Glacier.

Relative chronology. To develop a relative chronology of drift sheets alongside Transantarctic outlet glaciers, we used a combination of geologic features (surface morphology, moraine pattern, and crosscutting relationships), surface boulder weathering, and soil development (depth of staining, cohesion, visible salts, ghosts, maximum color development, morphogenetic salt stage, and weathering stage). First, we differentiated drift sheets in icefree areas alongside Beardmore (Denton et al., 1989), Hatherton (Bockheim et al., 1989), and upper Taylor Glaciers (Fig. 10)
(Bockheim, 1982). Second, we used soil development to determine relative ages of drifts among these outlet glaciers. We feel justified in this approach because drifts alongside these outlet glaciers are all composed of the same parent material (dolerite and sandstone gravel), occur near the polar plateau in an ultraxerous to xerous cold desert climate, and have similar topography (moraine crest) and elevation (1500$1800 \mathrm{~m}$ ).

Table 2 and Figure 11 compare five soil morphologic features on drifts alongside upper Taylor, Hatherton, and Beardmore Glaciers. Depth of staining appears to be the most useful relative-age discriminator, followed by depth of coherence and depth of visible salts (Fig. 11). Although surface boulder weathering features are useful in distinguishing among drift sheets in a particular area, these features are not helpful in correlating drifts among areas. On the basis of analysis of variance there are few significant differences in soil properties on the Britannia I, Britannia II, and Beardmore drifts. There also are few differences in these properties on the Danum and Meyer drifts. "Taylor II"/Bonney drift has 


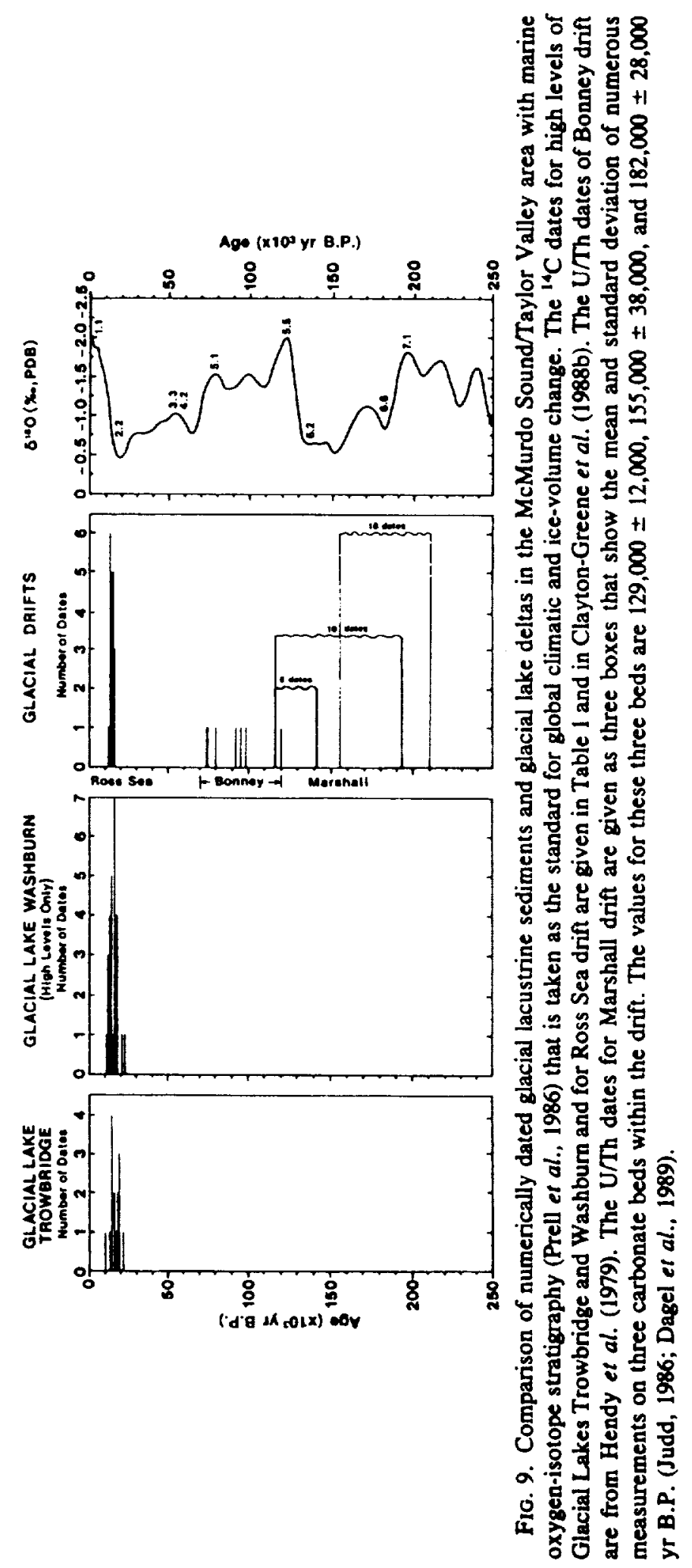




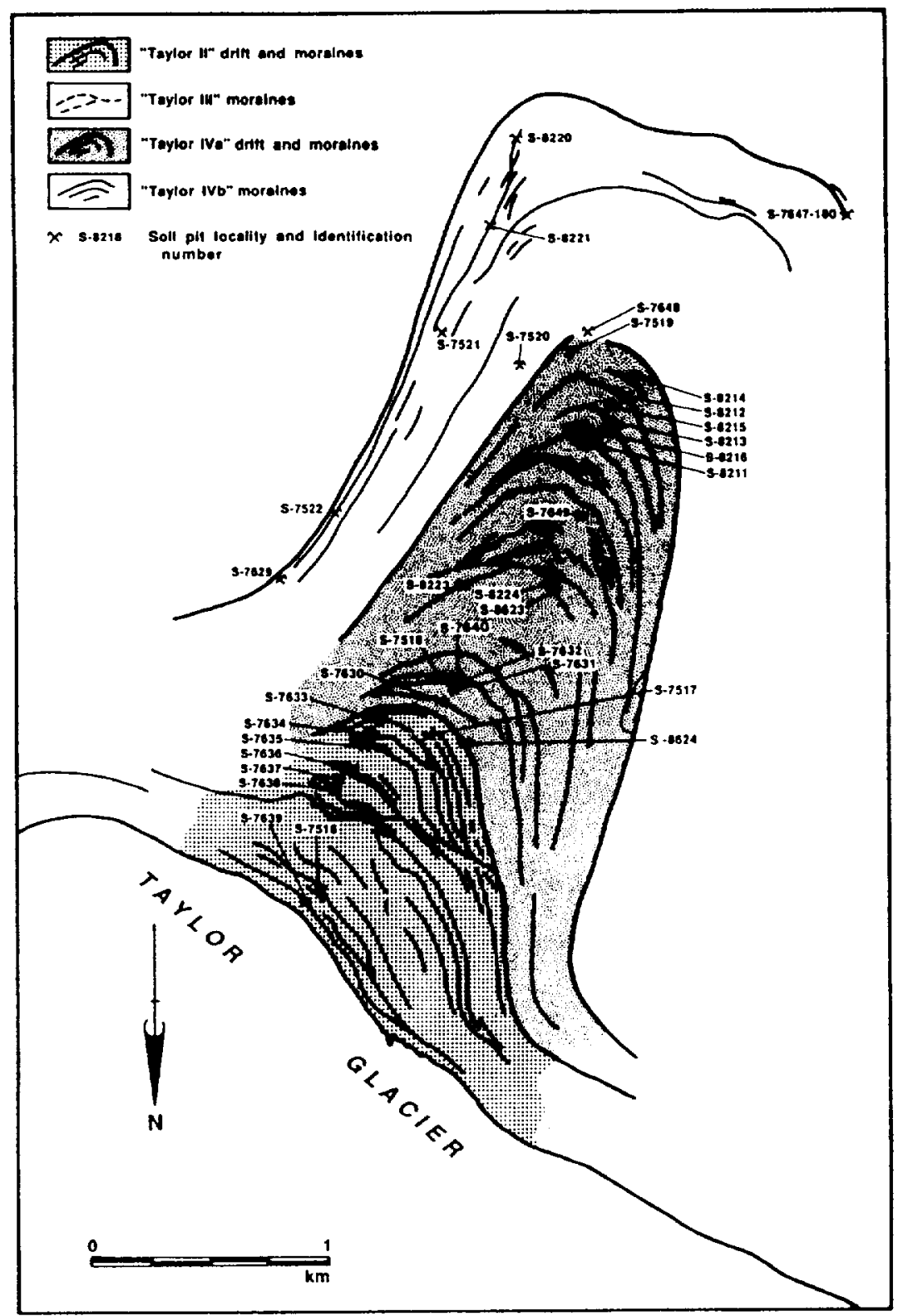

Fig. 10. Drift sheets and moraines in Arena Valley beside upper Taylor Glacier. See Figure 8 for location. Map base from vertical aerial photograph. Drift names in quotes are temporary.

soil properties intermediate between the drifts of these two groups. Soil development is greatest on "Taylor III" drift.

These correlations are consistent with the geologic interpretations of the respective drift sheets. The Beardmore/Britannia and Meyer/Danum drift sets both produce longitudinal profiles of Transantarctic outlet glaciers that reflect grounded ice deep in the Ross Embayment. In contrast, the Bonney drift occurred between grounding epi- sodes in McMurdo Sound and the adjacent Ross Embayment, and the "Taylor III" drift occurred prior to the grounding episode reflected by Meyer/Danum drift (Table 3).

Linkage of numerical and relative chronologies. Essential to our analysis of the Ross ice drainage system is the linkage of numerically dated drift sheets to the relative drift chronology derived from Figure 11. Bonney drift is prominent is both chronologies. Unfortunately, Ross Sea and 
Table 2. Morphological Soll Properties on Drifts in the Beardimore, Hatherton, and McMurdo Sound/Taylor Valley Areas of the Transantarctic Mountains*

\begin{tabular}{lcccccccc}
\hline \multicolumn{1}{c}{ Drift } & $\begin{array}{c}\text { Isotopic } \\
\text { stage }\end{array}$ & $\begin{array}{c}\text { No. of } \\
\text { profiles }\end{array}$ & Staining & Coherence & Matrix salts & Ghosts & C.D.E. \\
\hline Britannia I & 2 & 9 & $2 \mathrm{~d}$ & $6 \mathrm{~b}$ & $0 \mathrm{c}$ & $<$ le & $6 \mathrm{~d}$ \\
Britannia II & 2 & 10 & $5 \mathrm{~cd}$ & $5 \mathrm{~b}$ & $0 \mathrm{c}$ & $7 \mathrm{bcd}$ & $10 \mathrm{c}$ \\
Beardmore & 2 & 41 & $6 \mathrm{c}$ & $6 \mathrm{~b}$ & $0 \mathrm{c}$ & $1 \mathrm{c}$ & $7 \mathrm{~d}$ \\
Bonney & 5 & 11 & $8 \mathrm{~b}$ & $9 \mathrm{~b}$ & $0 \mathrm{c}$ & $3 \mathrm{cde}$ & $19 \mathrm{a}$ \\
Danum & 6 & 10 & $10 \mathrm{~b}$ & $26 \mathrm{a}$ & $8 \mathrm{~b}$ & $12 \mathrm{~b}$ & $13 \mathrm{~b}$ \\
Meyer & 6 & 30 & $10 \mathrm{~b}$ & $22 \mathrm{ab}$ & $9 \mathrm{ab}$ & $7 \mathrm{c}$ & $10 \mathrm{c}$ \\
"Taylor III" & 7 & 8 & $22 \mathrm{a}$ & $29 \mathrm{a}$ & $12 \mathrm{a}$ & $21 \mathrm{a}$ & $17 \mathrm{a}$ \\
\hline
\end{tabular}

a Color development equivalents (from Buntley and Westin, 1965).

* Values within a column followed by the same letter are not different at $\boldsymbol{P} \leqslant 0.05$ based on Fisher's PLSD.

Marshall drifts cannot be tied into the relative chronology by using soil development because of distinct differences in drift composition among the pertinent drift sheets. Therefore, we use other arguments that involve geometric relations of drift sheets.

In Taylor Valley, Bonney drift is crosscut by Ross Sea drift (Fig. 8). This relativeage relationship is consistent with numerical ages for Bonney (Stage 5) and Ross Sea (Stage 2) drifts. Further, the lack of an intermediate-age drift implies that Bonney drift is younger than Marshall drift. Such a relative-age relation is supported by numerical ages for Ross Sea (Stage 2), Bonney (Stage 5), and Marshall (Stage 6) drifts. Soil development shows Britannia I and II drifts to be younger than Bonney drift (Fig. 11). This makes Ross Sea drift the most likely correlative of the Britannia drifts in the Mc-
Murdo Sound/Taylor Valley area. Such a correlation is consistent with the fact that Britannia and Ross Sea drifts are both taken to represent major grounding in this portion of the Ross Embayment. Further, from geologic considerations we argue that the Britannia drifts represent one major glacial event. Finally, available ${ }^{14} \mathrm{C}$ dates suggest that Britannia drift limits are late Wisconsin in age. For these reasons, we correlate Britannia II drift with the older portion of Ross Sea drift and Britannia I with the younger portion of Ross Sea drift. This means that Britannia I drift represents a readvance superimposed on general recession from the Britannia II drift limit. Such a correlation is discussed in detail elsewhere in this issue (Bockheim et al., 1989).

The major weakness of correlating Britannia II and outer Ross Sea drift is that we

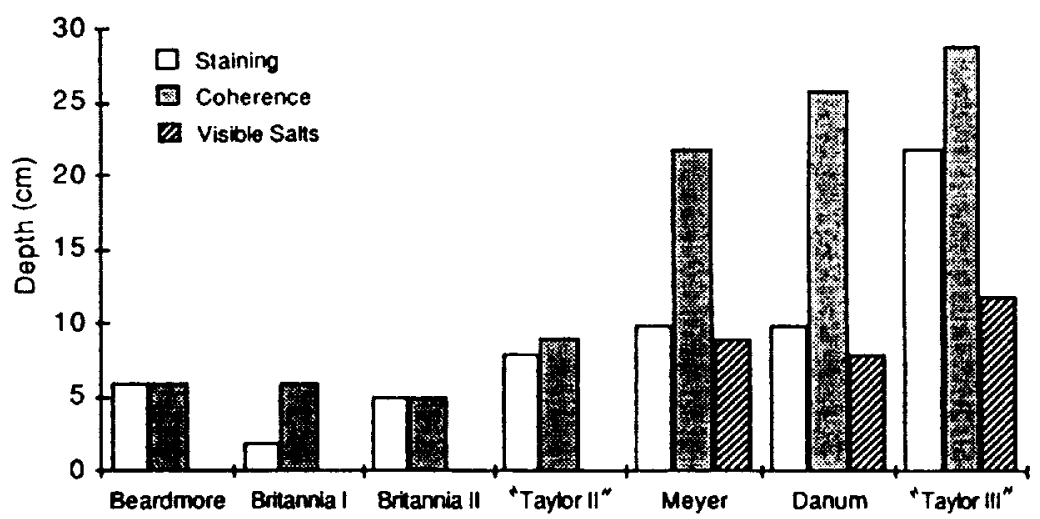

FIG. 11. Comparisons of soil morphologic properties among drift sheets alongside Taylor ("'Taylor II"/"Taylor III"), Hatherton (Britannia I, Britannia II, and Danum), and Beardmore (Beardmore, Meyer) Glaciers (Fig. 1). 
Table 3. Suggested Correlations of late Quaternary Drift Sheets in the Beardmore Glacier, hatherton Glacier, and McMurdo Sound/Taylor Valley areas of the

Transantarctic Mountains

\begin{tabular}{lccc}
\hline $\begin{array}{c}\text { Beardmore Glacier } \\
\text { (Denton et al., 1989) }\end{array}$ & $\begin{array}{c}\text { Hatherton Glacier } \\
\text { (Bockheim et al., 1989) }\end{array}$ & McMurdo Sound & Taylor Glacier \\
\hline Plunket drift & $\begin{array}{l}\text { Hatherton drift } \\
\text { Britannia I drift } \\
\text { Britannia II drift }\end{array}$ & Ross Sea drift & "Taylor I" drift \\
Beardmore drift & Danum drift & Marshall drift & "Taylor II"/Bonney drift \\
Meyer drift & "Taylor III" drift \\
\hline
\end{tabular}

have no control on soil development on sandstone-dolerite gravel drift numerically dated between isotope Stage 2 and Stage 6 in age. This weakness leaves open several alternative correlations. The first is that Britannia I drift correlates with outermost Ross Sea drift. This means that the Britannia II drift limit must represent a major grounding event that is younger than the Bonney advance and yet older and more extensive than Ross Sea grounding. A possible age for such a grounding event is that of isotope Stage 4. We think that this first alternative is implausible because it calls for a major grounding event near Hatherton Glacier that is not recognized in the McMurdo Sound/Taylor Valley area. Further, it is not in agreement with geologic considerations that Britannia I and II drifts cannot be separated in several areas alongside Hatherton Glacier (Bockheim et al., 1989). The second alternative is that both Britannia drifts are older than Ross Sea drift and yet younger than Bonney drift. We think that this second alternative is unlikely because it calls for two major grounding episodes near Hatherton Glacier that go unrecognized in the McMurdo Sound record. In addition, it means that the extensive late Wisconsin grounding episode recorded in McMurdo Sound by the Ross Sea drift goes unrecorded near Hatherton Glacier.

We now expand our stratigraphy of late Quaternary drifts along the length of the Transantarctic Mountains. Table 3 illustrates our discussion. We start by comparing in Figure 9 our numerical drift chronol- ogy with the marine oxygen-isotope record taken as the standard of late Quaternary climate and ice-volume change on a global scale. In Figure 9 it is evident that the outer portion of Ross Sea drift is of late Wisconsin age and correlates with marine isotope Stage 2, the last global ice-sheet maximum. By our relative chronology, the outer limits of Beardmore and Britannia drifts are thought to correlate with outer Ross Sea drift from geological arguments given previously. On the basis of the agreement of field mapping results from the Beardmore Glacier area (Mercer, 1972; Denton et al., 1988), we think it is very likely that Mercer's (1972) correlation of Reedy III and Beardmore III (our Beardmore) drifts is correct. We also follow Stuiver et al. (1981) in equating the "younger" drift at Terra Nova Bay with Ross Sea drift at McMurdo Sound. This correlation is based on surface weathering characteristics, morphologic drift form, limiting ${ }^{14} \mathrm{C}$ ages, and relativeage relationship to emerged Holocene beaches. In Terra Nova Bay and McMurdo Sound, these drifts both represent the youngest grounding episode, have wellpreserved surface morphology, commonly exhibit sharp outer limits, have minimum ${ }^{14} \mathrm{C}$ ages of $7020-6600$ yr B.P. (see below) for final ice recession, and are cut by emerged Holocene beaches (see below).

In Figure 9 Marshall drift corresponds with marine isotope Stage 6, representing the penultimate global glaciation. By our relative chronology, Marshall drift correlates with Danum drift at Hatherton glacier 
and Meyer drift at Beardmore Glacier. Finally, Figure 9 shows that Bonney drift correlates with marine isotope Stage 5 .

\section{Ice-Sheet Configuration}

We use the upper limits of Ross Sea, Britannia II, Beardmore, Reedy III, and "younger" Terra Nova Bay drifts to reconstruct the late Wisconsin (Stage 2) configuration of the Ross ice drainage system. We assume our stratigraphy is correct. Another assumption is that these drift limits are strictly synchronous. This second assump- tion can only be tested by new numerical dating techniques. However, we recognize that the complex interactions of sea level and climate can lead to asynchronous drift limits during an individual glaciation.

We give two extreme reconstructions of the areal extent and surface elevations of the Ross ice drainage system at the height of late Wisconsin glaciation (Figs. 12a and $12 b)$. Neither is based on ice-flow models, nor do we use seafloor sediments because they lack numerical chronology. Rather, both reconstructions are fixed by our outlet

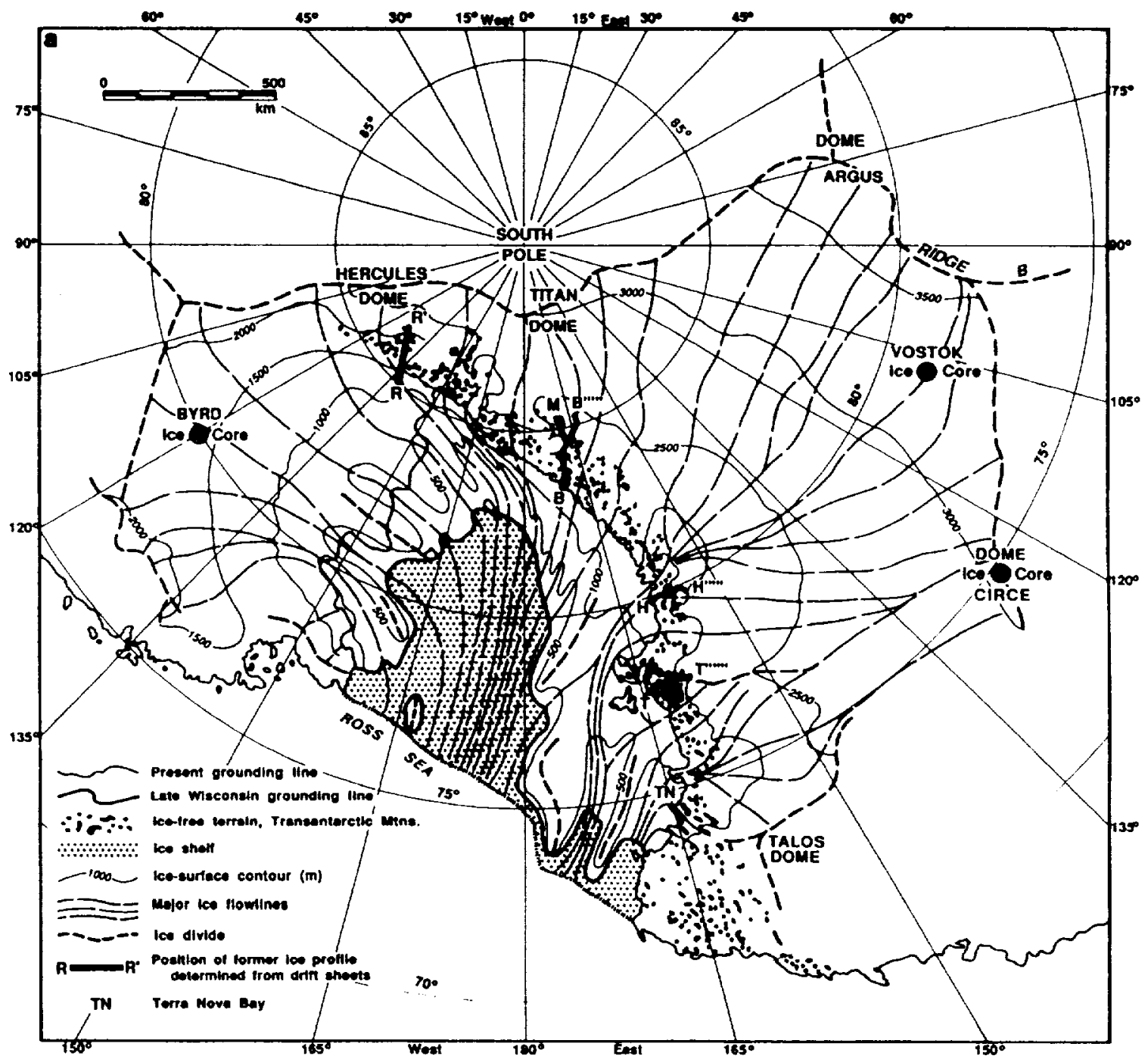

Fig. 12. Minimum (a) and maximum (b) reconstructions of grounded late Wisconsin ice in the Ross Embayment permitted by glacial geologic data from the Transantarctic Mountains. Isostatic compensation is not considered in these reconstructions. The position of the ice-shelf front shown in both diagrams is arbitrary. It is possible that shelf ice extended well offshore at the height of late Wisconsin glaciation. Lettered sections show positions of outlet glacier profiles in Figures 2, 3, 4, and 6. 


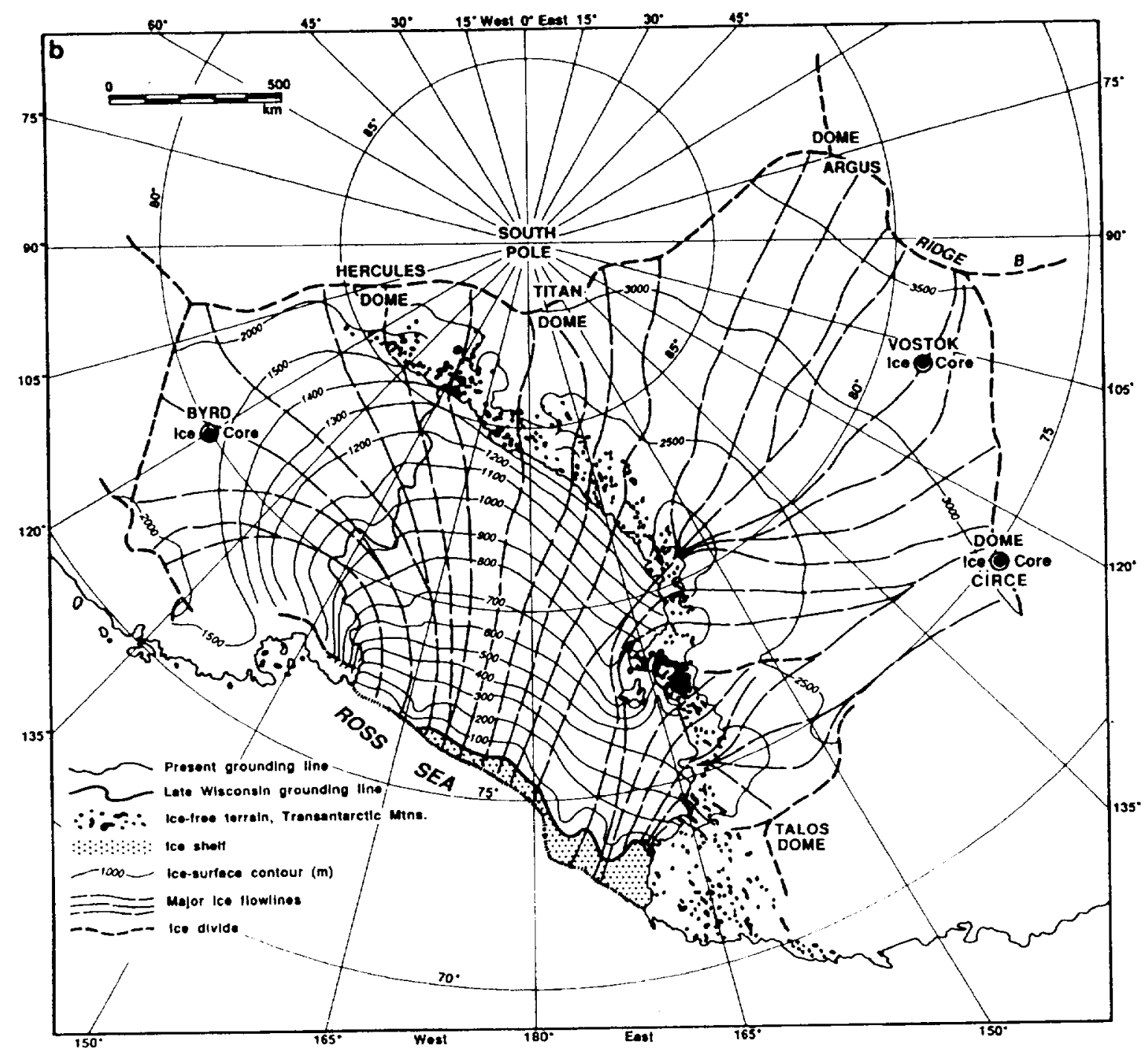

FIG. 12-Continued

glacier profiles, by the surface elevation of grounded ice in McMurdo Sound, by controlling ${ }^{14} \mathrm{C}$ dates on the extent of Taylor Glacier and, by implication, the height of the McMurdo Dome. As a result, both reconstructions depict grounded ice along the entire inner Ross Embayment and both show little change of the inland plateau surface adjacent to the Transantarctic Mountains between Taylor and Reedy Glaciers. The two reconstructions are identical in interior portions of the drainage system in East and West Antarctica. There we reproduce elevations similar to today's because of uncertainties about whether the inland surface increased or decreased slightly due to the interplay of sea-level and precipita- tion effects (this paper; Denton et al., 1989; Alley and Whillans, 1984). The major difference lies in the areal extent of grounded ice in the outer Ross Embayment and is due to differing glaciological assumptions. The minimum reconstruction is based on the assumption that West-Antarctic-type ice streams persisted in the Ross Embayment at the last glacial maximum and hence that the surface slope of ice flowing from West Antarctica did not change significantly. Under this assumption, the Transantarctic Mountains data, particularly from Reedy and Beardmore Glaciers, limit seaward extent of the late Wisconsin grounding line. The resulting reconstruction shows a deep extension of shelf ice into the central Ross 
Embayment (Fig. 12a). The maximum reconstruction is possible only if WestAntarctic-type ice streams did not exist in the Ross Embayment during late Wisconsin glaciation. This could occur if initial grounding-line advance was accompanied by coeval northward expansion of the Ross Ice Shelf to near the continental shelf edge, where it could ground extensively on submarine banks. The interior Ross Embayment could then fill by ice inflow from West Antarctica and from thickening Transantarctic outlet glaciers. The result, shown in Figure $12 \mathrm{~b}$, would be a thickening, slowmoving slab of grounded ice in the Ross Embayment that never achieved equilibrium conditions and that lacked throughflowing ice streams.

\section{Climate}

Late Wisconsin climate in the Ross ice drainage system is inferred from a combination of ice-core and geologic data. Jouzel et al. (1989) inferred from isotope records in the Dome Circe and Vostok ice cores (Fig. 1) that late Wisconsin full-glacial climate lasted from 25,000 to 15,000 yr B.P. Mean annual surface temperature is inferred to have been $9^{\circ} \mathrm{C}$ colder than now, and annual accumulation $50 \%$ less than now. Similar results come from the Byrd ice core in West Antarctica (Fig. 1) (Jouzel et al., 1989).

Glacial geologic evidence from the Transantarctic Mountains bears on the interpretations of paleoclimate from isotope records of interior ice cores. One critical assumption of such interpretations is that the interior plateau ice surface was no higher than now at the late Wisconsin glacial maximum. Otherwise, parts or all of the isotope records may simply reflect changes in ice-surface elevation. Direct control on ice-sheet elevation could come from total gas content of the ice cores themselves (Lorius et al., 1985), but the validity of this technique has been questioned (Paterson and Hammer, 1987). Our outletglacier profiles form an alternative control on interior ice elevations during late Wis- consin time, for they occur on strategic ice flowlines that lead back to interior domes and ice-core sites (Fig. 1). The Reedy III, Beardmore, and Britannia II profiles are consistent in indicating that the interior polar plateau near these flowlines probably did not rise more than $35-100 \mathrm{~m}$ during late Wisconsin (Stage 2) time. In fact, the glacial geologic evidence does not preclude a slight lowering along these flowlines, particularly inland of Beardmore Glacier. Taylor Glacier was no larger than now during late Wisconsin time. In fact, almost certainly its ice surface declined and its snout receded. This suggests little change, and probably a slight lowering, of the McMurdo Dome inland from Taylor Valley on the East Antarctic plateau. In summary, these glacial geologic data indicate little change of inland ice surfaces along critical ice flow lines that lead to major interior domes and pass near the important Vostok and Dome Circe ice cores, as well as little change of the McMurdo Dome. Hence, a significant rise in the interior ice surface can almost certainly be eliminated as a factor in interpreting the isotopic record at Dome Circe and Vostok in terms of temperature decrease during late Wisconsin time.

Is the reduced full-glacial precipitation inferred from ice cores consistent with the glacial geologic record in the Transantarctic Mountains? This record shows recession of Taylor Glacier and local alpine glaciers concurrent with full expansion of grounded Ross Sea ice during late Wisconsin time (Stuiver et al., 1981). Further, Rutkowski Glacier in the Dominion Range was behind its present terminal position when Beardmore Glacier thickened to its late Wisconsin limit (Denton et al., 1989). Finally, the plateau surface along flowlines inland of our outlet-glacier profiles showed little change, and may have declined, at the late Wisconsin maximum. Together, these glacial events can all be explained by reduced late Wisconsin precipitation in the Transantarctic Mountains, an interpretation consistent with the inferences drawn from isotope records in ice cores. 
There is, however, one aspect of the glacial geologic record that may be at variance with the paleoclimatic interpretation from ice cores. From our previous discussion, it is obvious that large lakes formed in the Dry Valleys during late Wisconsin time. For example, Glacial Lake Washburn occupied Taylor Valley (Stuiver et al., 1981; Denton et al., 1988) (Fig. 8) and Glacial Lake Trowbridge existed in Miers Valley (Clayton-Greene et al., 1988b). Our preliminary mapping results in other ice-free valleys indicate that such lakes were widespread and not restricted solely to Taylor and Miers Valleys.

We do not yet know why such lakes existed during the last glacial maximum. Several explanations are possible and each should be tested thoroughly. The first is that subglacial outflow from beneath Ross Sea ice lobes fed these lakes (Stuiver et al., 1981). We no longer favor this alternative. A second explanation is that input came solely from surface glacier melt. The increased volume of meltwater associated with higher lake levels could have come from increased intensity of melt, or from a longer melt season, or from both factors. There are several variations on this scenario. The increased volumes of meltwater necessary for Glacial Lake Trowbridge and Glacial Lake Washburn in the Fryxell basin could have come solely from the surface of Ross Sea ice lobes projecting into Miers and Taylor Valleys. But such an explanation cannot apply to the Bonney basin of Glacial Lake Washburn. Here late Wisconsin lake levels rose to the mid-valley threshold without any overflow from the Fryxell basin. This suggests that increased volume of meltwater from local glaciers, as well as from Ross Sea ice lobes, is almost certainly an important contributing factor to high late Wisconsin lake levels. In such a case, the increased meltwater input could have resulted simply from warmer late Wisconsin temperatures (Denton et al., 1985). Such postulated warming could have been widespread, in which case there is a serious discrepancy with temperature inferences drawn from all three major ice cores in the Ross ice drainage system. Such widespread warming also disagrees with our inferences that late Wisconsin retraction of local glaciers resulted from widespread aridification in the Transantarctic Mountains. Or the temperature increase could have been confined to the Dry Valleys. Clayton-Greene et al. (1988b) suggested that one local cause of such warming might have been a decrease in katabatic winds. We do not find this suggestion appealing, for we think that continued katabatic winds during late Wisconsin time were necessary to ablate Ross Sea glacier lobes flowing into ice-free valleys.

An alternative solution to the glacial-lake problem depends on the sensitivity to snow cover of meltwater production in the icefree valleys. For example, meltwater production in the Dry Valleys can be decreased dramatically by late spring snowstorms (Chinn, 1981). Widespread grounded and shelf ice in the Ross Sea, along with lower ice-age temperatures, could have promoted the aridification in the Dry Valleys and elsewhere along the Transantarctic Mountains inferred from shrinkage of small glaciers and little change in the inland plateau surface. Coastal storms would no longer have penetrated the Dry Valleys. Such intense aridification would have resulted in little or no snow cover in the Dry Valleys through the entire sunlight season. Together with the low-albedo ablation zone of the Ross Sea ice sheet in McMurdo Sound and in eastern Taylor Valley (due to surface volcanic debris), this aridification could have decreased the regional albedo and, in the absence of snowfall, the length of the melt season. This explanation for increased lake levels is not in conflict with lower late Wisconsin regional temperatures and accumulation.

\section{HOLOCENE STAGE}

\section{General Statement}

Glacial geologic evidence indicates that grounded ice receded from the western Ross Embayment at the end of late Wiscon- 
sin and beginning of Holocene time. Simultaneous expansion occurred for local alpine glaciers and for Taylor Glacier. Isotopic records from the Vostok and Dome Circe ice cores are interpreted in terms of climatic warming and increased accumulation beginning abruptly about 15,000 yr B.P. and continuing into early Holocene time (Lorius et al., 1984, 1985; Jouzel et al., 1987, 1989). We review here our current knowledge of the glacial history of the Transantarctic Mountains during the late Wisconsin/early Holocene transition.

\section{Radiocarbon Chronology}

Ross Sea and equivalent drifts. In the previous section we argued that the distal portions of Ross Sea, Britannia II, Beard- more, Reedy III, and the "younger" drift at Terra Nova Bay date to the late Wisconsin maximum. We now review available ${ }^{14} \mathrm{C}$ dates of the proximal portions of these drift sheets. Such dates give the timing of grounded ice recession from the western and southwestern Ross Embayment. We start in the north at Terra Nova Bay.

In the Terra Nova Bay region Adamussium colbecki shells from a recent moraine at the edge of the floating Nansen Ice Sheet afforded an age of $7020 \pm 60 \mathrm{yr}$ B.P. (QL174) (Table 1; Fig. 13). This date is minimum for grounded ice recession from the sample site and hence minimum for the "younger" drift.

On the west coast of McMurdo Sound, several ${ }^{14} \mathrm{C}$ dates pertain to proximal Ross
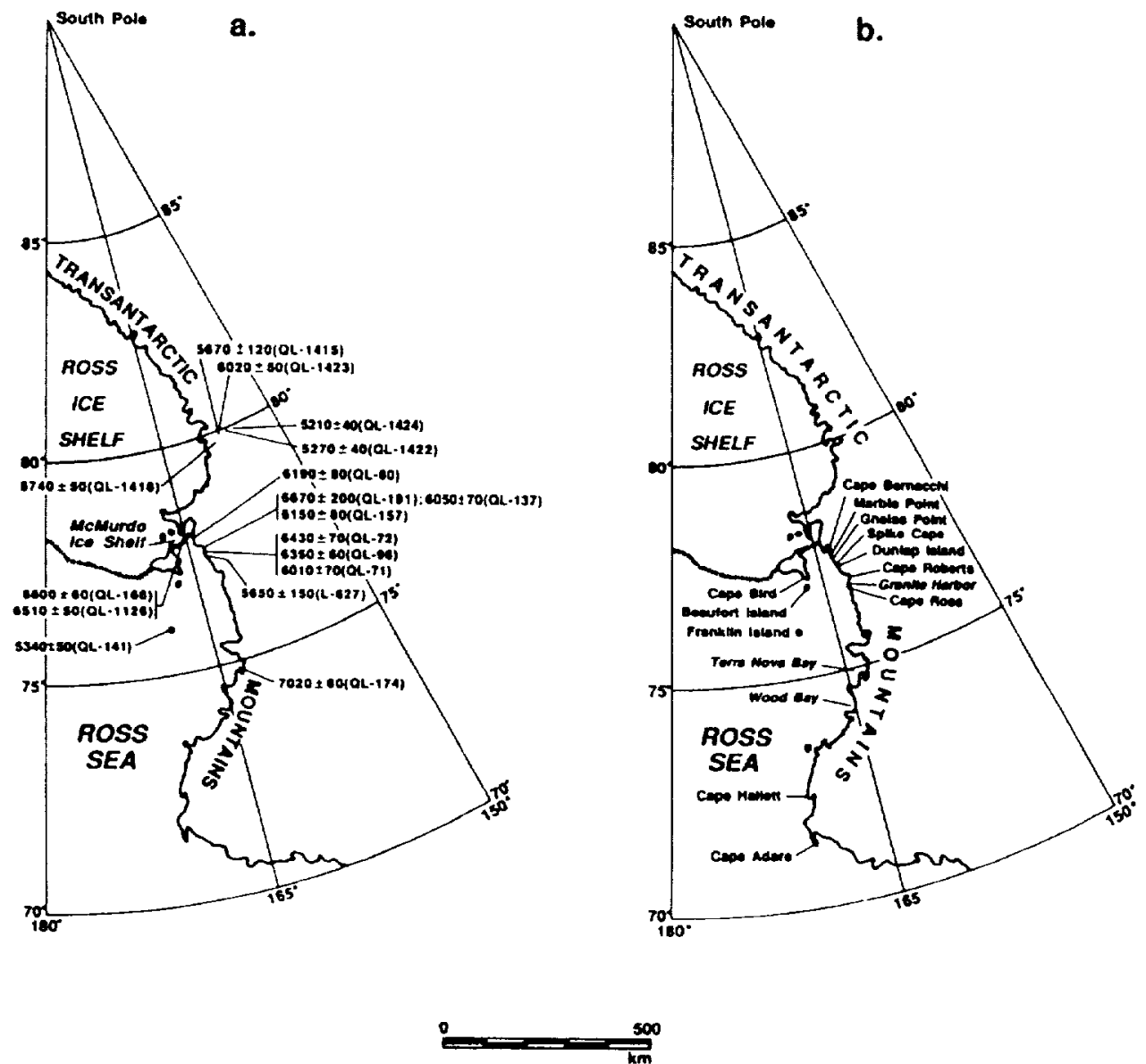

Fig. 13. Selected ${ }^{14} \mathrm{C}$ dates of late Wisconsin/Holocene recession of grounded ice from the western Ross Embayment (a); locations of raised beaches in the western Ross Embayment (b). 
Sea drift (Table 1; Fig. 7). In Taylor Valley a date of 13,040 \pm 190 yr B.P. (QL-1569) for blue-green algae in glacial lacustrine sediments affords a minimum age for recession of the Ross Sea glacial lobe from the valleymouth threshold near Explorers Cove. A similar sample on the seaward slope of this valley-mouth threshold gives a minimum age for ice recession from the sample site of $11,370 \pm 120$ yr B.P. (QL-1914). A lacustrine delta near the top of this threshold dates to $12,420 \pm 130$ yr B.P. (QL-1913) (Fig. 8), giving a minimum age for ice recession from the sample site. Lacustrine deltas on the same seaward slope of the threshold record a lake dammed between the threshold and the retreating Ross Sea ice. ${ }^{14} \mathrm{C}$ dates of blue-green algae in these deltas indicate that grounded Ross Sea ice still occupied McMurdo Sound near Taylor Valley as late as $8900 \pm 60 \mathrm{yr}$ B.P. (QL1393) and $8340 \pm 120$ yr B.P. (QL-993) (Fig. 5).

A complex of small deltas rests on Ross Sea drift on the south wall of Ferrar Glacier valley near McMurdo Sound. These deltas postdate the maximum of Ross Sea ice extent and hence afford minimum ${ }^{14} \mathrm{C}$ ages for Ross Sea drift (Table 1; Fig. 5). Dates of two deltas at 43 and $62 \mathrm{~m}$ elevation are $10,020 \pm 40$ yr B.P. (QL-1036) and $9860 \pm$ 160 yr B.P. (QL-995), respectively. Along the west coast of McMurdo Sound near the Royal Society Range, minimum ages for lowering of the Ross Sea ice surface to less than $100 \mathrm{~m}$ above present sea level are 9490 $\pm 140 \mathrm{yr}$ B.P. (Y-2399) and $12,500 \pm 40 \mathrm{yr}$ B.P. (QL-1590) on samples of blue-green algae. Finally, a date of $6190 \pm 80$ yr B.P. (QL-80) on blue-green algae is minimum for ice recession to the present coastline.

Further south in the Transantarctic Mountains, ${ }^{14} \mathrm{C}$ dates of blue-green algae deposited in small lakes perched beside the receding Hatherton Glacier afford minimum ages for ice-surface lowering (Figs. 4 and 13; Bockheim et al., 1989). They indicate lowering of the Hatherton Glacier surface to near its present position by $5210 \pm$ $40 \mathrm{yr}$ B.P. (QL-1424) in its upper reaches,
$5670 \pm 120$ yr B.P. (QL-1415) in its middle reaches, and $6020 \pm 50$ yr B.P. (QL-1423) in its lower reaches. Lower Darwin Glacier near the Ross Ice Shelf achieved its current surface elevation by $5740 \pm 50$ yr B.P. (QL1418).

Emerged marine deposits. Emerged beach deposits occur along the west coast of the Ross Embayment from Cape Bernacchi in McMurdo Sound northward to Cape Adare, as well as at Cape Bird and on Beaufort and Franklin Islands in the Ross Sea (Fig. 13). Locations of emerged beaches shown in Figure 13 are from Nichols (1968), Stuiver et al. (1981), and Mabin (1986). All these emerged beaches are $35 \mathrm{~m}$ or lower in elevation (Nichols, 1968; Stuiver et al., 1981; Mabin, 1986). These beaches are taken to represent isostatic rebound consequent on recession of grounded ice from the western Ross Embayment. Although these emerged beaches are consistent with the former presence of grounded ice in the western Ross Embayment, they are not a straightforward indicator of former ice thickness for two reasons. First, the beaches are largely undated and thus cannot afford isobases of uplift. Second, the highest beach in an individual area may simply reflect uplift since recession of floating shelf ice rather than of grounded ice (Denton et al., 1988).

There are few ${ }^{14} \mathrm{C}$ dates associated with these beaches. One relates directly to a beach at Marble Point ( $5650 \pm 150$ yr B.P.: L-627) (Nichols, 1968). Others are of associated deltas at Marble Point $(6010 \pm 70 \mathrm{yr}$ B.P., QL-71; and $6430 \pm 70$ yr B.P., QL-72) and bedded colluvium at Franklin Island (5340 \pm 50 yr B.P., QL-141) (Table 1; Figs. 5 and 13).

Emerged marine deposits occur at the mouth of Taylor Valley at the edge of Explorers Cove despite the absence of welldefined beaches. Numerous ${ }^{14} \mathrm{C}$ dates of $A$. colbecki valves come from these deposits or from deep within a marine delta at the valley mouth. These dates are minimum for incursion of marine waters into Explorers Cove in early Holocene time. The oldest 
such dates are $6050 \pm 70$ yr B.P. (QL-137), $6150 \pm 80$ yг B.P. (QL-157), and $6670 \pm 200$ yr B.P. (QL-191). Together with the ${ }^{14} \mathrm{C}$ ages of lacustrine deltas on the seaward slope of the valley-mouth threshold, these dates indicate recession of grounded Ross Sea ice from this sector of McMurdo Sound between 8340 and 6670 yr B.P.

McMurdo Ice Shelf. The McMurdo Ice Shelf, an extension of the Ross Ice Shelf, now covers southern McMurdo Sound and contains evidence that bears on deglacial history of grounded ice from the Ross Embayment (Stuiver et al., 1981). Some debris bands on the shelf surface are remnant from the Ross Sea Ice Sheet (Stuiver et al., 1981); they show that the ice shelf formed in part by thinning of the grounded Ross Sea Ice Sheet in McMurdo Sound. Other debris bands formed in Holocene time by basal freezing and seaward flow. One such band that extends northward from Black Island to the shelf edge displays progressively greater ${ }^{14} \mathrm{C}$ ages of shells and barnacles from south to north. The oldest samples at the northern tip of this band afforded ages of $6510 \pm 50 \mathrm{yr}$ B.P. (QL-1126) and $6600 \pm$ 60 yr B.P. (QL-166) (Table 1; Figs. 5 and 13) (Stuiver et al., 1981). Hence, Holocene grounding-line recession of the Ross Sea Ice Sheet had reached Black Island by 6600 yr B.P. The McMurdo Ice Shelf extended farther north until recently, thereby explaining the absence of emerged beaches south of Cape Bird on Ross Island and south of Cape Bernacchi along the west coast of McMurdo Sound (Stuiver et al., 1981).

Taylor Glacier and alpine glaciers. Taylor Glacier and alpine glaciers in Taylor Valley both occupy their maximum positions since late Wisconsin time, with the exception of a minor fluctuation shown by scattered ice-cored moraines of Holocene age. The evidence comes largely from ${ }^{14} \mathrm{C}$ dated perched deltas of Glacial Lake Washburn (Fig. 8) near Taylor Glacier that were discussed previously. Rhone Glacier is now overriding deltas dated to $12,700 \pm 190 \mathrm{yr}$ B.P. (QL-1709) and $16,470 \pm 250$ yr B.P.
(QL-1046); Hughes Glacier terminus is close to deltas dated to $18,170 \pm 70$ yr B.P. (QL-1137) and $18,830 \pm 80$ yr B.P. (QL1248); and Canada Glacier terminus is adjacent to a delta dated to $14,300 \pm 300 \mathrm{yr}$ B.P. (QL-1385). Commonwealth Glacier rests on Ross Sea glacial lacustrine drift dated nearby to $15,660 \pm 60$ yr B.P. (QL$1140) ; 13,970 \pm 300$ yr B.P. (QL-1793); $12,130 \pm 300$ yr B.P. (QL-1794); 14,470 \pm 330 yr B.P. (QL-1795); $14,730 \pm 150$ yr B.P. (QL-1156); and $13,700 \pm 400$ yr B.P. (QL1234). That this situation is not unique to Taylor Valley is shown by several alpine glaciers along the west coast of McMurdo Sound that have advanced into Ross Sea drift or over emerged Holocene beaches.

Glacial lakes. In Taylor Valley high levels of Glacial Lake Washburn in the Bonney basin persisted above the mid-valley threshold until $12,700 \pm 190$ yr B.P. (QL1709), and perhaps until $11,820 \pm 70$ yr B.P. (QL-1576). In the Fryxell basin they persisted above the valley-mouth threshold until at least $12,450 \pm 350$ yг B.P. (QL-1043) (Fig. 8). Hence, we conclude that a Ross Sea glacier lobe plugged eastern Taylor Valley until this time. We know from ${ }^{14} \mathrm{C}$ dates given earlier that the Ross Sea glacier lobe in eastern Taylor Valley had retreated from the crest of the valley-mouth threshold by 13,040 yr B.P. and from its seaward slope by 11,370 yr B.P. (Fig. 8). Nevertheless, higher-than-present lake levels persisted in the Fryxell basin as late as $9200 \pm$ 40 yr B.P. (QL-1142) (Stuiver et al., 1981), although such levels were much lower than the valley-mouth threshold. One probable water source for this late-lingering lake was westward overflow through a channel on the valley-mouth threshold from a lake trapped between the threshold and a grounded Ross Sea ice sheet in Explorers Cove. Such a trapped lake existed until at least $8340 \pm 120$ yr B.P. (QL-993), the age of the lowest lacustrine delta on the seaward slope of the threshold. This icedammed lake had ceased to exist by $6670 \pm$ 200 yr B.P. (QL-191), the age of the oldest marine shells in Explorers Cove. Hence, 
we infer that grounded Ross Sea ice cleared Explorers Cove between 8340 and $6670 \mathrm{yr}$ B.P. (Fig. 13).

The most striking feature highlighted by the ${ }^{14} \mathrm{C}$ dates in Taylor Valley is that high lakes in the Fryxell and Bonney basins were common during the entire span of late Wisconsin time but were almost absent during Holocene time. The exception is the late-lingering, but relatively low, lake that lasted in Fryxell basin until 9200 yr B.P.

\section{Late Wisconsin/Early Holocene Ice-Sheet Changes}

In summary, we infer from our data that the grounded Ross Sea ice sheet receded from the western Ross Embayment in late Wisconsin and early Holocene time. Radiocarbon dates in Marshall and Taylor Valleys indicate that Ross Sea glacier tongues had receded eastward nearly into McMurdo Sound by $12,500-13,040$ yr B.P., but ${ }^{14} \mathrm{C}$ dates from Ferrar and Taylor Valleys imply that grounded ice lingered in western McMurdo Sound until 10,020 to 8340 yr B.P. Grounded Ross Sea ice had cleared the coast of the western and southern Ross Embayment by 7020 yr B.P. at Terra Nova Bay (Stuiver et al., 1981); 6430, 6670, 6600, 6190 , and 7750 yr B.P. at McMurdo Sound (Stuiver et al., 1981); and 5210, 5670, 6020, and $5750 \mathrm{yr}$ B.P. at Hatherton Glacier (Bockheim et al., 1989).

Our geologic evidence shows strikingly different behavior of the plateau surface of the East Antarctic Ice Sheet inland of the Transantarctic Mountains. No massive decrease in surface elevation occurred here in late Wisconsin/Holocene time comparable with recession of grounded ice from the Ross Embayment. The Reedy III, Beardmore, and Britannia II profiles show that the decline of the upper reaches of the Reedy, Beardmore, and Hatherton Glaciers during the late Wisconsin/Holocene transition was only $30-100 \mathrm{~m}$. In fact, these ice profiles even allow the possibility that the plateau surface along inland flowlines could be higher now than that during late Wisconsin time. In accord with this, Taylor Glacier and local alpine glaciers have expanded so that they are now at, or very close to, their maximum positions since late Wisconsin time. Likewise Rutkowski Glacier, which drains an independent ice cap on the Dominion Range near the upper reaches of Beardmore Glacier, has advanced at least $2.5 \mathrm{~km}$ over Beardmore drift and is now at its most robust configuration since late Wisconsin time.

\section{Climate}

Holocene surface climate in the Ross ice drainage system is inferred from a combination of ice-core and geologic data. Isotope records from the Vostok and Dome Circe ice cores are taken to show a marked warming that terminated late Wisconsin glacial climates. The ice-core chronology, based on an ice-flow model tied to accumulation, places the beginning of this climatic warming at about 15,000 yr B.P. The full amplitude of temperature change across the late Wisconsin/Holocene transition is taken to be $9^{\circ}-10^{\circ} \mathrm{C}$, and the Holocene accumulation rate is interpreted to be about twice the full-glacial value (Jouzel et al., 1989).

Glacial geologic evidence bears on these interpretations of paleoclimates from interior ice cores. First, our outlet glacier profiles show little change, and permit even a slight rise, of the inland plateau. The strong implication is that the isotopic change in interior ice cores across this same transition reflects widespread climatic change and not simply massive lowering of the interior plateau ice surface. The expansion of Taylor Glacier and local alpine glaciers, as well as the advance of Rutkowski Glacier at the head of Beardmore Glacier, during the late Wisconsin/Holocene transition is best explained by increased accumulation in the Transantarctic Mountains and on the polar ice plateau just inland (Denton et al., 1971, 1989). This, too, is in accord with inferences on increased interior accumulation drawn from ice cores.

A problem again arises in interpreting glacial lake history in the Dry Valleys in terms of a rise in temperature and accumu- 
lation through the late Wisconsin/Holocene transition. Extensive late Wisconsin lakes were succeeded by limited Holocene lakes generally even less extensive than those of today. Why would lakes decrease in both area and volume, if the climate warmed and precipitation increased? There are at least three explanations for this observed history of glacial lakes. First, the meltwater source from Ross Sea ice lobes disappeared with recession of grounded ice from McMurdo Sound. That this is partly responsible is shown by the lake-level history in eastern Taylor Valley. However, it does not explain the disappearance of lakes in the Bonney basin. The second explanation is that lake-level variations reflect strictly the effect of summer temperatures on glacier melt and hence on lake-level variation. This scenario disagrees with regional temperature changes inferred from ice cores. A third explanation involves both the recession of Ross Sea ice from McMurdo Sound and the decreasing aridity of the Dry Valleys through the late Wisconsin/Holocene transition. Ross Sea ice recession would remove a low-albedo, debris-laden ablation ice surface from McMurdo Sound, while simultaneously opening the western Ross Sea and removing a barrier to the penetration of storms. One result could be increased spring and summer albedo in McMurdo Sound from replacement of dirty glacial ice by clean sea ice and in the Dry Valleys from sporadic snowstorms. The overall effect could be to decrease the length of the melt season, while at the same time removing a meltwater source. These mechanisms could produce the observed lake-level history by climatic changes consistent with those inferred from ice cores.

\section{DISCUSSION}

The first recognition of a former grounded great ice sheet in the Ross Embayment came early in this century (Scott, 1905; David and Priestley, 1914). Within the last decade there appeared maximum and minimum reconstructions for late Wisconsin glacial conditions that attempted to explain available geological and glaciological data (Stuiver et al., 1981, pp. 375-380). It is now apparent that both reconstructions are flawed (Denton et al., 1988). For example, the CLIMAP maximum reconstruction (Stuiver et al., 1981, p. 375) shows ice elevations that were too high in the southern Ross Embayment and over West Antarctica, where they conflict with interpretations drawn from the total gas content of the Byrd ice core (Raynaud and Whillans, 1982) and radio-echo layers in the West Antarctic Ice Sheet (Whillans, 1976). The CLIMAP minimum reconstruction, on the other extreme, is essentially the same as today's ice sheet in the Ross Embayment; this conflicts with glacial geologic evidence from Darwin/Hatherton and Beardmore Glaciers (Bockheim et al., 1989; Denton et al., 1989). Interim reconstruction designed to reconcile some of these problems (Drewry, 1979; Hughes et al., 1985; Denton et al., 1986, 1988) also have deficiencies (Denton et al., 1988).

Figure 12 gives our new minimum (a) and maximum (b) reconstructions for the Ross ice drainage system based on the same Transantarctic outlet profiles but on two differing glaciological assumptions discussed earlier. Our chronology and reconstructions highlight the difference in behavior of the continental and marine portions of the Ross ice drainage system (Fig. 14). During late Wisconsin maximum glaciation, the Ross Embayment partially or wholly filled with grounded ice, while elevations of interior plateau ice remained nearly unchanged and perhaps even lowered slightly. During the late Wisconsin/Holocene deglacial hemicycle, grounded ice receded from the inner Ross Embayment while local Transantarctic glaciers expanded and the inland plateau surface changed very little and may even have increased slightly in surface elevation.

What mechanisms could have caused this behavior difference of marine and inland portions of the Ross ice drainage system? We argue from our glacial geologic data that the fundamental cause for the wide- 


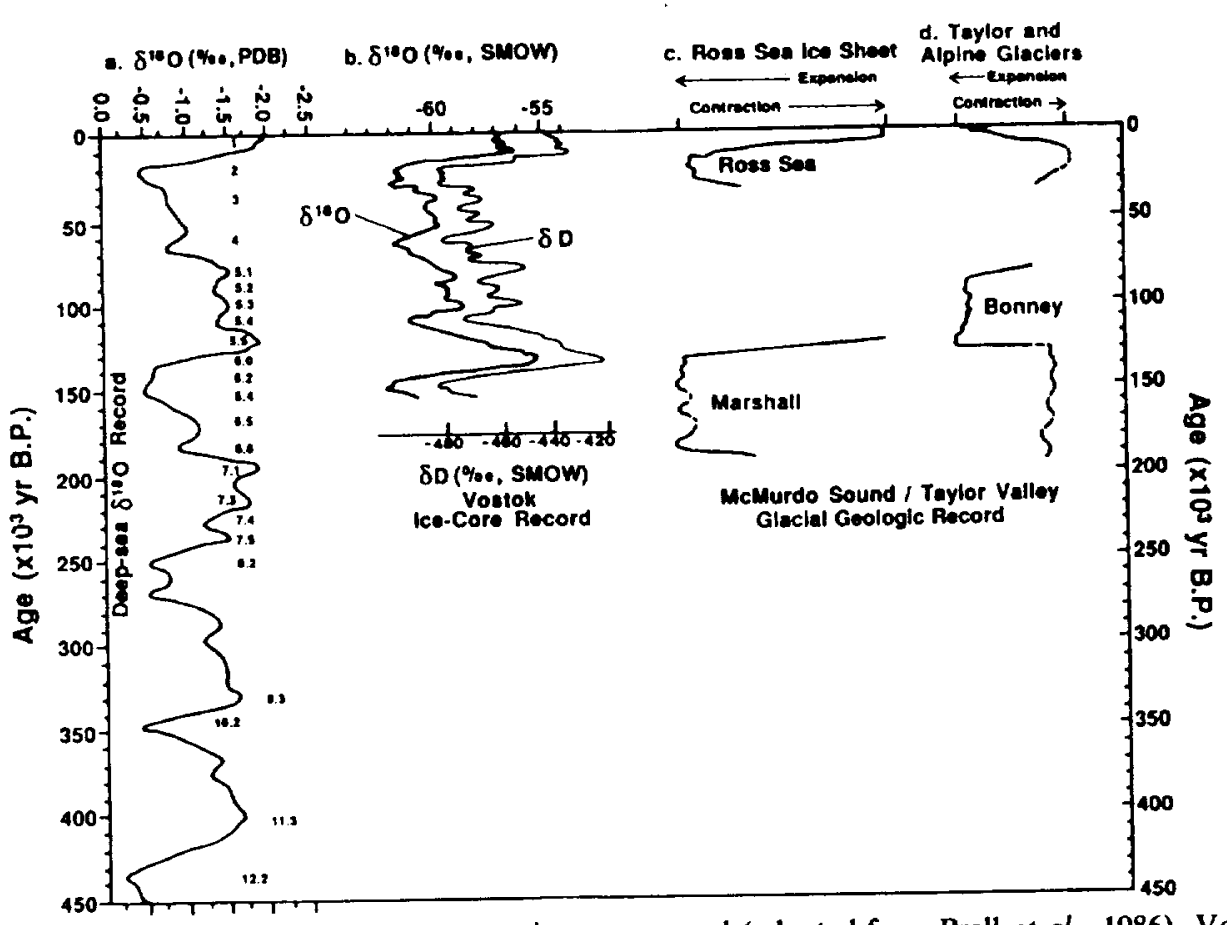

FiG. 14. Global average deep-sea oxygen-isotope record (adapted from Prell et al., 1986), Vostok ice-core oxygen-isotope and deuterium records (adapted from Lorius et al., 1985 and Jouzel et al., 1987; chronology from ice model and accumulation rates), and McMurdo Sound/Taylor Valley glacial geology record (chronology from numerical dates). The record of alpine glaciers in the right-hand column includes Taylor Glacier, which drains the McMurdo Dome (Drewry, 1980, 1982).

spread grounding shown in Figures $12 \mathrm{a}$ and $12 \mathrm{~b}$ must be sought in mechanisms that affected the Ross Embayment and not the inland plateau ice. We make this argument because, in view of decreased precipitation and little change in plateau elevations, it is unlikely that greater flow of inland ice through Transantarctic outlet glaciers caused grounding in the Ross Embayment. Therefore, such grounding must represent the response of the Ross Ice Shelf to reduced temperature, precipitation, and sea level. We agree with Hollin (1962) that sea-level variations, caused by Northern Hemisphere ice sheet fluctuations, are an important control on grounding-line variation and on pinning and unpinning the ice shelf. Grounding-line advance, accompanied by increased pinning of the ice shelf, could also cause northward migration of the calving front to the edge of the continental shelf, where the ice shelf could ground on relatively shallow submarine banks. This, in turn, could stimulate widespread grounding farther south. Ice-age temperature low- ering may also aid ice-shelf grounding through decreased basal melting and increased protection of the calving front by pack ice. Both of these factors would operate solely in the Ross Embayment and could together overcome reduced precipitation to cause widespread grounding. We also argue from geologic evidence that the slight changes of the East Antarctic inland plateau and inland West Antarctica reflect the interplay between grounding and precipitation effects.

Changes of the marine and inland portions of the Ross ice drainage system correlate closely with the last and penultimate glacial/interglacial cycles (Fig. 14). Our identification of differing primary controls in these two portions of the drainage system is consistent with such behavior. The potential interdependence of these primary controls can explain the tight out-of-phase behavior that is so closely related to global glacial/interglacial cycles (Fig. 14). For example, low ice-age temperatures, which can enhance grounding in the Ross Embay- 
ment, are closely tied to reduced precipitation (Lorius et al., 1985). Likewise, lower ice-age sea level promotes grounding in the Ross Sea, which removes a local moisture source and reduces inland penetration of storms.

The behavior of the Ross ice drainage system has global relevance to late Wisconsin sea level and climate. The maximum CLIMAP reconstruction illustrates thicker and more extensive grounded ice in the Ross Embayment and in West Antarctica (Stuiver et al., 1981, Fig. 7-26) than we now show in Figures $12 \mathrm{a}$ or $12 \mathrm{~b}$. These two new reconstructions illustrate that the contribution of the Ross ice drainage system to sea-level lowering would depend on the relative magnitude of ice-volume loss (water equivalent) in the inland portion to volume gain (water equivalent above buoyancy) in the marine portion. Depending on the reconstruction eventually accepted for each portion, the sea-level contribution could be close to zero. If such a conclusion can be generalized, it means that the maximum CLIMAP reconstruction overestimated the Antarctic contribution to late Wisconsin sea-level fall (Denton and Hughes, 1981).

\section{CONCLUSIONS}

(i) From soil development, numerical dates, and glacial geologic data, we correlate Reedy III drift (Reedy Glacier), Beardmore drift (Beardmore Glacier), Britannia drifts (Hatherton and Darwin Glaciers), Ross Sea drift (McMurdo Sound/Taylor Valley), and "younger" drift (Terra Nova Bay). From 51 pertinent ${ }^{14} \mathrm{C}$ dates in Table 1 and an additional 15 from Clayton-Greene et al. (1988b), we conclude that Ross Sea drift is late Wisconsin (isotope Stage 2) in age.

(ii) From ice-surface profiles based on these drift sheets, we produce two reconstructions of the Ross ice drainage system at the height of late Wisconsin (isotope Stage 2) glaciation. Both show little elevation change of the interior polar plateau. The major differences occur in the extent of grounded ice in the Ross Embayment. One reconstruction shows limited grounded ice, whereas the other shows grounded ice to be extensive but thin. Both reconstructions show late Wisconsin ice-volume increase in the Ross ice drainage system to be considerably less than depicted by the maximum CLIMAP reconstruction (Denton and Hughes, 1981).

(iii) Radiocarbon dates from the western Ross Embayment show that late Wisconsin/early Holocene recession of grounded ice was underway by $13,040 \mathrm{yr}$ B.P. and was complete by $6020-6600 \mathrm{yr}$ B.P. Little change occurred in the East Antarctic plateau adjacent to the Transantarctic Mountains during massive recession of grounded ice from the Ross Embayment.

(iv) Sea-level variation and ice-shelf basal melting probably controlled grounding in the Ross Embayment. Precipitation variations and the grounding effect on Transantarctic Mountains outlet glaciers probably controlled minor elevation changes of the polar plateau adjacent to the Transantarctic Mountains.

(v) The Vostok and Dome Circe ice cores in the Ross ice drainage system are considered premier recorders of far-southern paleoclimate (Jouzel et al., 1989). The Transantarctic Mountains outlet glacier profiles are consistent with this conclusion, in that they preclude an alternative explanation that the isotope records from these ice cores simply reflect extensive elevation changes of the East Antarctic polar plateau.

\section{ACKNOWLEDGMENTS}

The Antarctic field work and laboratory research at the University of Maine and the University of Wisconsin were funded by the Division of Polar Programs of the National Science Foundation. The Quaternary Isotopes Laboratory at the University of Washington is supported by the National Science Foundation. Logistic support in Antarctica was afforded by the National Science Foundation and the United States Navy. H. B. Conway, B. Hess, J. E. Leide, and R. Weed aided in the field work. T. J. Hughes gave valuable insights about ice-sheet reconstructions and lake-level fluctuations. This manuscript benefited greatly from reviews by T. J. Hughes, M. L. Prentice, J. F. 
Splettstoesser, and D. E. Sugden. R. Kelly drafted the maps and longitudinal sections. $N$. Kealiher processed the manuscript.

\section{REFERENCES}

Alley, R. B., and Whillans, I. M. (1984). Response of the East Antarctic Ice Sheet to sea-level rise. Journal of Geophysical Research 89, 6487-6493.

Bockheim, J. G. (1982). Properties of a chronosequence of ultraxerous soils in the Trans-Antarctic Mountains. Geoderma 28, 239-255.

Bockheim, J. G., Wilson, S. C., Denton, G. H., Andersen, B. G., and Stuiver, M. (1989). Late Quaternary ice-surface fluctuations of Hatherton Glacier, Transantarctic Mountains. Quaternary Research 31, 229-254.

Buntley, G. J., and Westin, F. C. (1965). A comparative study of developmental color in a ChestnutChernozem-Brunizem soil climosequence. Soil Science Society of America Proceedings 29, 579-582.

Chinn, T. J. H. (1981). Hydrology and climate in the Ross Sca area. Journal of the Royal Society of New Zealand 11, 373-386.

Clayton-Greene, J. M. (1986). "Proglacial sedimentation of late Wisconsin age in Miers Valley, Antarctica." M. Sci. thesis, University of Waikato, Hamilton.

Clayton-Greene, J. M., Hendy, C. H., and Denton, G. H. (1988a). The formation of debris mounds on Glacial Lake Miers. Antarctic Journal of the United States, in press.

Clayton-Greene, J. M., Hendy, C. H., and Hogg, A. G. (1988b). The chronology of a Wisconsin-aged proglacial lake in the Miers Valley, Antarctica. New Zealand Journal of Geology and Geophysics, in press.

Dagel, M. A., Hendy, C. H., Judd, F., Denton, G. H., and Stuiver, M. (1989). Stratigraphy and chronology of Stage 6 and 2 glacial deposits, Marshall Valley, Antarctica. Boreas, in press.

David, T. W. E., and Priestley, R. E. (1914). "Glaciology, Physiography, Stratigraphy, and Tectonic Geology of South Victoria Land: British Antarctic Expedition, 1907-1909. Vol. 1. Reports on the Scientific Investigations, Geology.

Debenham, F. (1921). "Recent and Local Deposits of McMurdo Sound Region: London, British Museum, British Antarctic (Terra Nova) Expedition (1910).' Vol 1, No. 3, pp. 63-90. Natural History Report. Geology.

Denton, G. H., and Armstrong, R. L. (1968). Glacial geology and chronology of the McMurdo Sound region. Antarctic Journal of the United States 3, 99 101.

Denton, G. H., Armstrong, R. L., and Stuiver, M. (1970). Late Cenozoic glaciation in Antarctica. Antarctic Journal of the United States 5, 15-22.

Denton, G. H., Armstrong, R. L., and Stuiver, M.
(1971). The late Cenozoic glacial history of Antarctica. In "The Lake Cenozoic Glacial Ages" (K. K. Turekian, Ed.), pp. 267-306. Yale Univ. Press, New Haven, CT.

Denton, G. H., Bockheim, J. G., Wilson, S. C., Leide, J. E., and Andersen, B. G. (1989). Late Quatemary ice-surface fluctuations of Beardmore Glacier, Transantarctic Mountains. Quaternary Research, 31, 183-209.

Denton, G. H., and Borns, H. W., Jr. (1974). Former grounded ice sheets in the Ross Sea. Antarctic Journal of the United States 9, 167.

Denton, G. H., Borns, H. W., Jr., Grosswald, M. G., Stuiver, M., and Nichols, R. L. (1975). Glacial history of the Ross Sea. Antarctic Journal of the United States 10, 160-164.

Denton, G. H., and Hughes, T. J. (1981). "The Last Great Ice Sheets." Wiley-Interscience, New York. Denton, G. H., and Hughes, T. J. (1983). Milankovitch theory of ice ages, hypothesis of ice-sheet linkage between regional insolation and global climate. Quaternary Research 20, 125-144.

Denton, G. H., Hughes, T. J., and Karlén, W. (1986). Global ice-sheet system interiocked by sea level. Quaternary Research 26, 3-26.

Denton, G. H., Prentice, M. L., and Burckle, L. H. (1988). Cenozoic History of the Antarctic Ice Sheet. In "The Geology of Antarctica" (R. Tingey, Ed.). Oxford Univ. Press, London, in press.

Denton, G. H., Stuiver, M., and Austin, K. (1985). Radiocarbon chronology of the last glaciation in the McMurdo Sound region. Antarctic Journal of the United States 19, 59-61.

Drewry, D. J. (1979). Late Wisconsin reconstruction for the Ross Sea region, Antarctica. Journal of Glaciology 24, 231-244.

Drewry, D. J. (1980). Pleistocene biomodal response of Antarctic ice. Nature (London) 287, 214-216.

Drewry, D. J. (1982). Ice flow, bedrock, and geothermal studies from radio-echo sounding inland of $\mathrm{Mc}$ Murdo Sound, Antarctica. In "Antarctic Geoscience” (C. Craddock, Ed.), pp. 977-983. Univ. of Wisconsin Press, Madison.

Drewry, D. J. (Ed). (1983). "Antarctica: Glaciological and Geophysical Folio." Scott Polar Research Institute, Cambridge.

Fillon, R. H. (1975). Late Cenozoic paleooceanography of the Ross Sea, Antarctica. Geological Society of America Bulletin 86, 839-845.

Hendy, C. H., Healy, T. R., Rayner, E. M., Shaw, J., and Wilson, A. T. (1979). Late Pleistocene glacial chronology of the Taylor Valley, Antarctica, and the global climate. Quaternary Research 11, 172-184.

Hollin, J. T. (1962). On the glacial history of Antarctica. Journal of Glaciology 4, 173-195.

Hughes, T. J., Denton, G. H., and Fastook, J. L. (1985). Is the Antarctic Ice Sheet an analogue for Northern Hemisphere paleo-ice sheets? In "Models 
in Geomorphology" (J. J. Woldenburg, Ed.), pp. 25-72. Allen and Urwin, Boston.

Jouzel, J., Lorius, C., Petit, J. R., Genthon, C., Barkov, N. I., Kotlyakov, V. M., and Petrov, V. M. (1987). Vostok ice core: A continuous isotopic temperature record over the last climate cycle (160,000 years). Nature (London) 329, 403-408.

Jouzel, J., Raisbeck, G., Benoist, J. P., Yiou, F., Lorius, C., Raynaud, D., Petit, J, R., Barkov, N. I., Korotkevitch, Y. S., and Kotlyakov, V. M. (1989). A comparison of deep Antarctic ice cores and their implications for climate between 65,000 and 15,000 years ago. Quaternary Research 31, 135-150.

Judd, F. M. (1986). "The chronology of the Ross Sea II glaciation, an Antarctic glaciation of Illinoian age." M. Sci. thesis, University of Waikato, Hamilton.

Kellogg, T. B., Truesdale, R. S., and Osterman, L. E. (1979). Late Quaternary extent of the West Antarctic Ice Sheet: New evidence from Ross Sea cores. Geology 7, 249-253.

Lorius, C., Jouzel, J., Ritz, C., Merlivat, L., Barkov, N. I., Korotkevich, Y. S., and Kotlyakov, V. M. (1985). A 150,000 year climatic record from Antarctic ice. Nature (London) 316, 591-596.

Lorius, C., Raynaud, D., Petit, J. R., Jouzel, J., and Merlivat, L. (1984). Late glacial maximumHolocene atmospheric and ice thickness changes from Antarctic ice core studies. Annals of Glaciology 5, 88-94.

Mabin, M. C. G. (1986). The Ross Sea section of the Antarctic ice sheet at 18,000 yr B.P.: Evidence from Holocene sea-level changes along the Victoria Land coast. South African Journal of Science 82, 506508.

Mercer, J. H. (1968). Glacial geology of the Reedy Glacier area, Antarctica. Geological Sociely of America Bulletin 79, 471-486.

Mercer, J. H. (1972). Some observations on the glacial geology of the Beardmore Glacier area. In "Antarctic Geology and Geophysics" (R. J. Adie, Ed.), pp. 427-433. Universitetsforlaget, Oslo.

Nichols, R. L. (1968) Coastal geomorphology, McMurdo Sound, Antarctica. Journal of Glaciology 7 $449-478$.

Paterson, W. S. B., and Hammer, C. V. (1987). Ice core and other glaciological data. In: "North America and Adjacent Oceans During the Last Deglaciation" (W. F. Ruddiman and H. E. Wright, Jr., Eds.), pp. 91-109. Geological Society of America, Boulder, CO.

PEwE, T. L. (1960). Multiple glaciation in the McMurdo Sound region, Antarctica, a progress report. Journal of Geology 68, 489-514.

Prell, W. L., Imbrie, J., Martinson, D. G., Morley, J. J., Pisias, N. G., Shackleton, N. J., and Streeter, H. F. (1986). Graphic correlation of oxygen isotope stratigraphy: Application to the Late Quaternary. Paleoceanography 1, 137-162.

Raynaud, D., and Whillans, I. M. (1982). Air content of the Byrd core and past changes in the West Antarctic Ice Sheet. Annals of Glaciology 3, 269-273.

Ross, Captain Sir James Clark (1847). "A Voyage of Discovery and Research in the Southern and Antarctic Regions During the Years 1839-1843," Vols. 1 and 2. John Murray, London.

Scott, R. F. (1905). "The Voyage of the Discovery," Vols. 1 and 2. Scribner's, New York.

Stuiver, M., Denton, G. H., Hughes, T. J., and Fastook, J. L. (1981). History of the marine ice sheet in West Antarctica during the last glaciation, a working hypothesis. In "The Last Great Ice Sheets" (G. H. Denton and T. H. Hughes, Eds.), pp. 319-436. Wiley-Interscience, New York.

Stuiver, M., and Polach, H. S. (1977). Discussionreporting of ${ }^{14} \mathrm{C}$ data. Radiocarbon $19,355-363$.

Whillans, I. M. (1976). Radio-echo layers and the recent stability of the West Antarctic Ice Sheet. $\mathrm{Na}$ ture (London) 264, 152-155. 